\title{
Advanced Organic Light-Emitting Devices for Enhancing Display Performances
}

\author{
Chung-Chih Wu, Chieh-Wei Chen, Chun-Liang Lin, and Chih-Jen Yang
}

\begin{abstract}
Various advanced organic light-emitting devices (OLEDs) for enhancing performances of OLED displays, particularly active-matrix OLED displays, are reviewed in this paper. These include top-emitting OLEDs, inverted OLEDs, high-contrast OLEDs, microcavity OLEDs, and their combinations. How these device structures enhance display performances, such as color saturation, brightness or emission efficiency, contrast, and aperture ratio of pixels, etc., are discussed. The critical technical issues and the status of development associated with each device technology are reviewed.
\end{abstract}

Index Terms-Active-matrix displays, high-contrast devices, inverted devices, microcavity, organic light-emitting devices (OLEDs) and displays, top-emitting devices.

\section{INTRODUCTION}

$\mathbf{O}$ RGANIC electroluminescence was first observed in thick organic crystals sandwiched between electrodes in the 1960s [1], [2]. However, the high operating voltage of devices ( $\sim 1000$ V) back then limited its practical use. In 1987, Tang and VanSlyke reported the first practical organic light-emitting diodes (OLEDs) [3]. They introduced the effective heterojunction devices of small-molecular-weight materials, which were fabricated by thermal evaporation and gave low operating voltage $(<10 \mathrm{~V})$ and attractive device efficiency $(\sim 1 \%$ photon/electron). Later in 1989, Tang, Van Slyke, and Chen further boosted the device efficiency by two to three times with the emissive-doping technique [4]. Such a technique also provides a facile way to tune the emission color simply by varying the guest dopants [4]. Not long after reports of OLEDs of small molecular weight, in 1990 the group of Cambridge University demonstrated polymer LEDs (PLEDs) with conjugated polymers [5]. In contrast with of vacuum processing of small-molecule OLEDs, the polymer layers in PLEDs were fabricated by spin-coating or other solution-casting processes.

\footnotetext{
Manuscript received July 16, 2005; revised September 9, 2005. This work was supported in part by the National Science Council of Republic of China and by the Electronic Research and Science Organization in the Industry Technology Research Institute (ERSO/ITRI). The work of C.-W. Chen and C.-L. Lin was supported by a MediaTek Fellowship.

C.-C. Wu is with the Department of Electrical Engineering, Graduate Institute of Electro-optical Engineering, and Graduate Institute of Electronics Engineering, National Taiwan University, Taipei 10617, Taiwan, R.O.C. (e-mail: chungwu@cc.ee.ntu.edu.tw).

C.-W. Chen, C.-L. Lin, and C.-J. Yang are with the Graduate Institute of Electro-optical Engineering, National Taiwan University, Taipei 10617, Taiwan, R.O.C.

Digital Object Identifier 10.1109/JDT.2005.858942
}

Since then, OLEDs have been subjects of intensive studies due to their various merits for flat-panel display applications. The distinguished characteristics of OLEDs for displays include high efficiency, wide viewing angle, fast response, and potentially low cost. In addition, their low processing temperatures, and thus versatility in substrates, render them suitable for some novel display applications, such as flexible displays. Over the past decade, the OLED display technology has made rapid progress, and various types of OLED displays have been demonstrated or commercialized. In 1997, Pioneer Corporation of Japan introduced the first OLED display product, which was a monochrome (green) passive-matrix OLED display with a moderate resolution [6]. Since then, passive-matrix OLED displays of various colors, area colors, and full colors were subsequently developed and commercialized by various companies. With further advance of OLED display technologies toward even higher image quality (e.g. higher resolutions, more colors, higher brightness etc.), higher efficiency, and larger areas, the passive-matrix architecture can no longer meet the requirements and the active-matrix architecture, which integrates OLEDs with transistor memory/driving circuits in each display pixel, has become the technology of choice [7]-[14].

High-performance active-matrix OLED displays (AMOLEDs) in general impose requirements in various aspects of the technology, including OLED materials with higher efficiencies and longer lifetimes [15]-[18], [81], designs of active-matrix circuits, providing better display performances (e.g., better uniformity, more grayscales, etc.) [7]-[14], and advanced OLED device structures that would give better optical and electrical performances (e.g., higher efficiencies, more saturated colors, higher contrast, lower voltage, etc.). In this article, we will focus on several advanced OLED structures that could enhance display performances of AMOLEDs: top-emitting OLEDs, inverted OLEDs, high-contrast OLEDs, and microcavity OLEDs.

The major technical issues associated with these advanced OLED structures in general are how to design their optical structures and how to prepare electrodes with required electrical and optical properties. Therefore, in this article, general discussions of the optical structures and optical characteristics of OLEDs are first given, and then used as a basis in discussing the optical characteristics of each advanced OLED structure, and how such characteristics are achieved with available electrode technologies. The paper then reviews the development of 


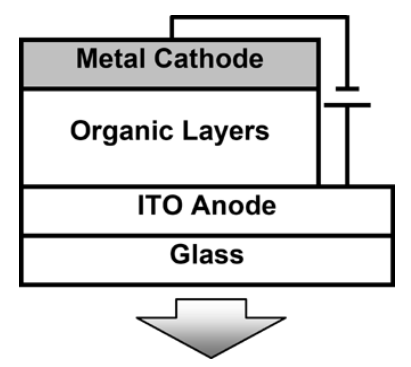

Fig. 1. Device structure of a conventional bottom-emitting OLED. (a)

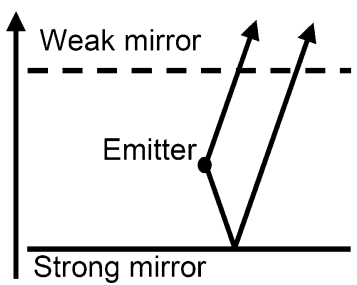

(b)

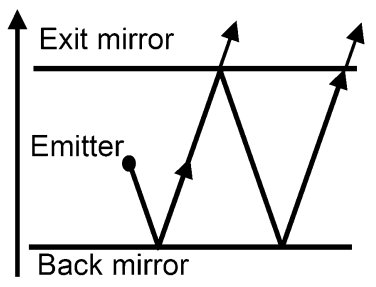

Fig. 2. Illustration of optical phenomena in OLEDs. (a) wide-angle interference in noncavity (weak-microcavity) OLED. (b) Wide-angle and multiple-beam interference in strong microcavity OLEDs.

these advanced OLED structures, starting with their technical purposes and then concentrating on major technical issues associated with each OLED technology. The discussions of each OLED technology then conclude with examples of applications in AMOLEDs. The paper is organized as follows. In Section II, the general theories and discussions of optical structures and characteristics of OLEDs are first given. Then four types of advanced OLED structure are discussed in sequence: top-emitting OLEDs in Section III, inverted OLEDs in Section IV, high-contrast OLEDs in Section V, and microcavity OLEDs in Section VI. Finally, Section VII concludes this paper.

\section{General Discussions of Optical STRUCtURES AND CHARACTERISTICS OF OLEDS}

The basic structure of OLEDs consists of multilayers of organic materials sandwiched between two electrodes (Fig. 1). The total thickness of organic materials is usually of the order of $100 \mathrm{~nm}$ and is comparable to the emission wavelength. Therefore emission properties of devices not only depend on intrinsic properties of emitting materials but also often are modified significantly by the optical structures of OLEDs. For instance, a typical OLED, which usually has one reflective metal cathode and one transparent indium tin oxide (ITO) anode on glass substrates (Fig. 1), behaves like a weak microcavity. The device exhibits wide-angle interference between directly emitting and reflected radiation, as represented in Fig. 2(a) [19], [20]. On the other hand, in some OLED structures, such as microcavity OLEDs and top-emitting OLEDs, etc., two electrodes of strong reflection are implemented into the devices, and one obtains a strong one-dimensional microcavity [19], [20]. The optical characteristics of such strong microcavity structure usually are significantly tailored not only by wide-angle interference but also by multiple-beam interference, as illustrated in Fig. 2(b)

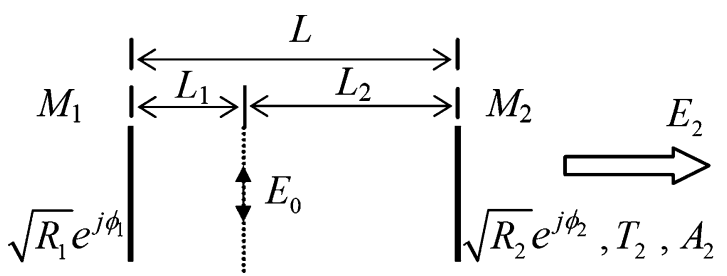

Fig. 3. Schematic diagram showing the general optical structure and characteristics of an OLED device.

[19], [20]. The optical microcavity effects, inherent with OLEDs, can spatially and spectrally redistribute the emission of devices, and therefore should be considered in any type of OLEDs. In this section, first a simpler and analytical formulation of micrcavity effects in OLEDs based on concepts of the Fabry-Perot cavity is given, which gives clearer physical insights but is limited to treatment of characteristics along the forward direction of devices. In the second part of this section, a brief description of rigorous electromagnetic modeling of optical characteristics of OLEDs is then given. Both will then be used as the basis in discussing optical characteristics of various OLED structures.

\section{A. Fabry-Perot Formulation}

Optically, an OLED may be considered as a Fabry-Perot cavity (either strong or weak cavity) embedded with a source, as shown in Fig. 3, in which $E_{0}$ represents the free-space electric-field intensity of the source, $E_{2}$ is the electric-field intensity of the wave out-coupled through the mirror $\mathrm{M}_{2}$, $\sqrt{ } R_{1} e^{j \phi 1}$ is the complex reflection coefficient of the mirror $\mathrm{M}_{1}$ (with a reflectance $\left.R_{1}\right), \sqrt{ } R_{2} e^{j \phi 2}$ is the complex reflection coefficient of the mirror $\mathrm{M}_{2}$ (with a reflectance, a transmittance and an absorptance of $R_{2}, T_{2}$ and $A_{2}$, respectively), $L_{1}$ is the source-to- $\mathrm{M}_{1}$ distance, $L_{2}$ is the source-to- $\mathrm{M}_{2}$ distance, and $L$ is the total cavity length $\left(L=L_{1}+L_{2}\right)$. In such a configuration, $\mathrm{M}_{1}$ and $\mathrm{M}_{2}$ are effective mirrors on two sides of the devices representing optical properties of interfaces and layer stacks beyond giving dominant reflection properties, while the total cavity length represents all other layers sandwiched between these two interfaces. Considering both the field redistribution due to cavity and the influence of cavity on the transition rate of molecular excited states, one obtains the enhancement factor $G_{\text {cav }}(\lambda)$ for emission through $\mathrm{M}_{2}$ (in the forward direction) relative to free-space emission at a wavelength $\lambda[21]-[24]$

$$
\begin{aligned}
& G_{\text {cav }}(\lambda) \\
& \quad=\frac{\left|E_{2}\right|^{2}}{\left|E_{0}\right|^{2}} \times \frac{\tau_{\text {cav }}}{\tau_{0}}=\left[1-\frac{4 \sqrt{R_{1}} \sin ^{2}\left(\frac{\phi_{1}-2 k L_{1}}{2}\right)}{\left(1+\sqrt{R_{1}}\right)^{2}}\right] \\
& \quad \times\left[\frac{T_{2}\left(1+\sqrt{R_{1}}\right)^{2}}{\left(1-\sqrt{R_{1} R_{2}}\right)^{2}+4 \sqrt{R_{1} R_{2}} \sin ^{2}\left(\frac{\phi_{1}+\phi_{2}-2 k L}{2}\right)}\right] \frac{\tau_{\text {cav }}}{\tau_{0}}
\end{aligned}
$$

$k$ is the wavevector in the organic layer, and $\tau_{\text {cav }}$ and $\tau_{0}$ are lifetimes of the molecular excited state in the cavity and in the free space, respectively. Similarly, one can also derive an equation similar to (1) for emission through the mirror $\mathrm{M}_{1}$ if it also 
permits optical transmission (e.g., in transparent OLEDs and double-side-emitting OLEDs).

Consider an organic emitter with an intrinsic emission spectrum $S(\lambda)$ which for convenience of following discussions is assumed Gaussian with a peak wavelength $\lambda_{\mathrm{em}}$ and a full-width at half-maximum (FWHM) $\Delta \lambda_{\mathrm{em}}$. According to (1), to maximize luminance through the mirror $\mathrm{M}_{2}$ of a cavity, it roughly requires to set resonance $\left(\phi_{1}+\phi_{2}-2 k L=2 m \pi, m\right.$ : an integer representing the cavity mode) near $\lambda_{\mathrm{em}}$ by adjusting the cavity length $L$ and to place the emitter near the antinode of $\mathrm{M}_{1}$ $\left(\phi_{1}-2 k L_{1}=2 l \pi, l\right.$ : integer). $G_{\text {cav }}(\lambda)$ in (1) describes the single-wavelength enhancement (in the forward direction) relative to free-space emission. Since organic emitters, in general, have rather broad emission spectra, of more practical interest, however, is the spectrally integrated enhancement $G_{\text {int }}$ for emission through one mirror of a particular OLED structure, as defined in (2), relative to emission from a conventional noncavity (indeed weak-microcavity) bottom-emitting OLED (Fig. 1) that has an ITO as the transparent bottom electrode and a high-reflectivity top metal electrode:

$$
G_{\mathrm{int}}=\frac{\int S(\lambda) G_{\mathrm{cav}}(\lambda) d \lambda}{\int S(\lambda) G_{\mathrm{con}}(\lambda) d \lambda}
$$

where $G_{\text {con }}(\lambda)$ is the emission enhancement of an optimized conventional bottom-emitting OLED (i.e., satisfy antinode and resonance conditions) relative to free-space emission, as described in (1). For such a conventional bottom-emitting OLED, typically one mirror has a strong reflectance of $\sim 90 \%$ (from highly reflective $\mathrm{Al}$ or $\mathrm{Ag}$ electrodes) and the other mirror has a weak reflectance of $\sim 3 \%$ (from the ITO-glass interface) and an absorptance of $\sim 0 \%$.

Consider an organic emitter with an intrinsic emission spectrum $S(\lambda)$ with $\lambda_{\mathrm{em}}$ of $520 \mathrm{~nm}$ and $\Delta \lambda_{\mathrm{em}}$ of $60 \mathrm{~nm}$. Fig. 4(a) shows $G_{\text {con }}(\lambda)$ of the optimized conventional bottom-emitting OLED for such an emitter, which is rather broad and remains rather constant over the spectral range of $S(\lambda)$. As a result, the emission spectrum from such a device (calculated by $S(\lambda) \times$ $G_{\text {con }}(\lambda)$ ) remains identical to $S(\lambda)$ [Fig. 4(b)]. On the other hand, Fig. 5(a) shows $G_{\text {cav }}(\lambda)$ (for emission through $\mathrm{M}_{2}$ ) of an optimized strong-microcavity OLED with $R_{1}=0.9, R_{2}=0.6$, and $A_{2}=0.1$ for the same emitter, which now has a much narrower shape and a much larger peak value than $G_{\mathrm{con}}(\lambda)$. As a result, the strong-micorcavity device shows narrowed emission (and thus better color purity) in comparison with the original intrinsic emission [Fig. 5(b)].

In general, for emission through one mirror (e.g., $\mathrm{M}_{2}$ ), $G_{\text {int }}$ is a function of $R_{1}, R_{2}, A_{2}\left(T_{2}=1-R_{2}-A_{2}\right)$, and $\Delta \lambda_{\text {em }}$ according to (1) and (2). Since $\tau_{\text {con }}$, the molecular excited-state lifetime in the conventional noncavity device in general differs from $\tau_{\text {cav }}, G_{\text {int }}$ also depends on the ratio $\tau_{\text {cav }} / \tau_{\text {con. }}$. Experimetally measured $\tau_{\text {cav }} / \tau_{\text {con }}$, however, usually is close to 1 (mostly 0.8-1) [23]-[27]. Thus for most purposes, it is good enough to set $\tau_{\text {cav }} / \tau_{\text {con }}$ to 1 for examining major influences of other device parameters. For instance, Fig. 6(a) shows calculated $G_{\text {int }}$ for the lowest cavity mode $(m=0, l=0)$ as a function of $R_{2}$ with $\Delta \lambda_{\mathrm{em}}=60 \mathrm{~nm}$ (and $\lambda_{\mathrm{em}}=520 \mathrm{~nm}$ for a typical OLED green emitter), $R_{1}=0.9-0.5$ and $A_{2}=0-0.25$. Results of Fig. 6(a) (a)

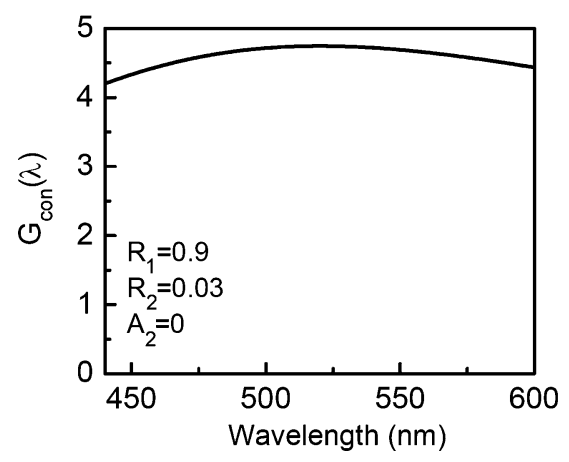

(b)

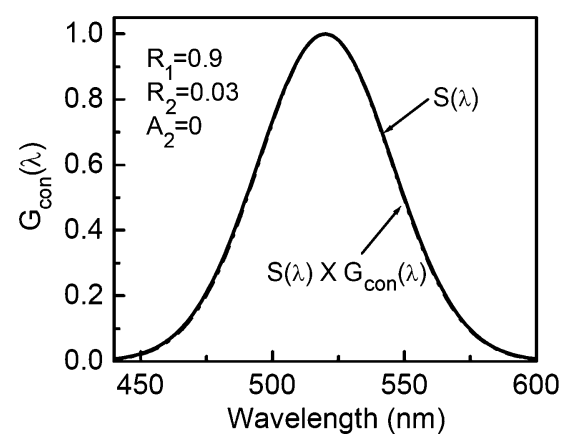

Fig. 4. (a) Forward enhancement factor for a conventional noncavity bottom-emitting OLED and (b) its effect on the device emission spectrum.

(a)

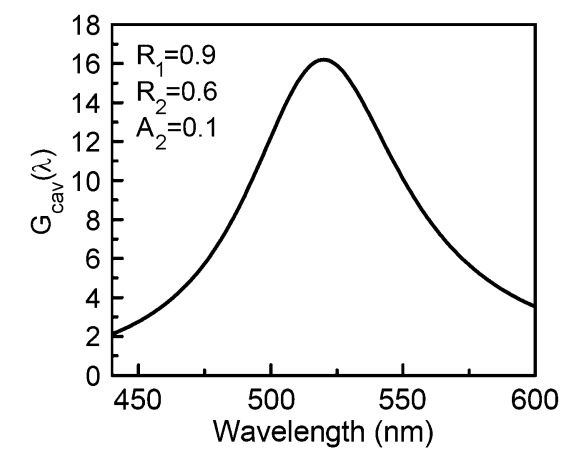

(b)

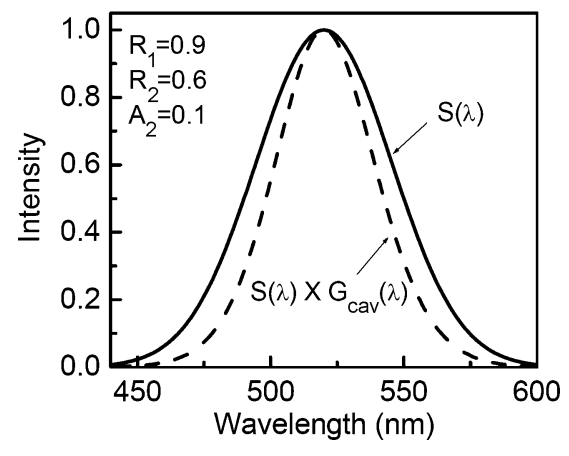

Fig. 5. (a) Forward enhancement factor for a microcavity OLED and (b) its effect on the device emission spectrum.

indicates that to obtain decent luminance (e.g. $G_{\text {int }}>1$ ) from one side of a particular device structure (e.g., through $\mathrm{M}_{2}$ here), it is critical to keep $R_{1}$ large enough and $A_{2}$ small enough, and have $\mathrm{M}_{2}\left(R_{2}\right)$ designed carefully. The maximal enhancement 
(a)

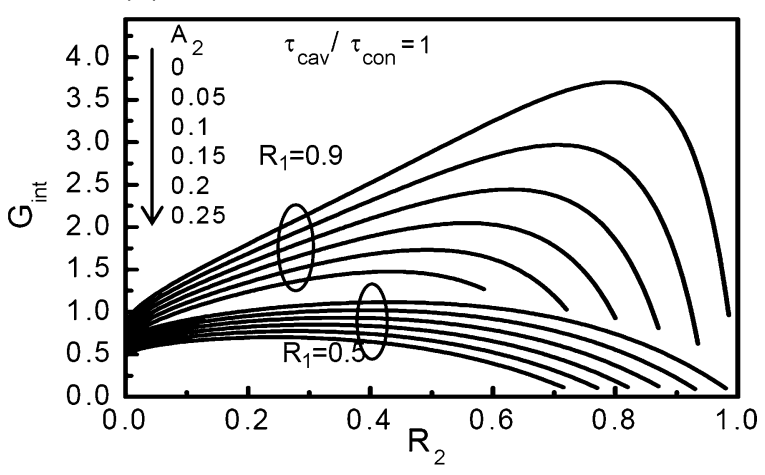

(b)

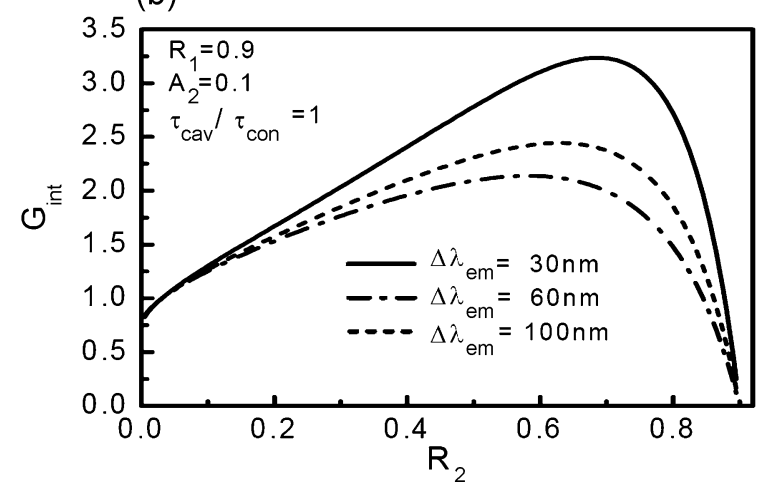

Fig. 6. Calculated $G_{\text {int }}$ as a function of $R_{2}\left(\lambda_{\text {em }}=520 n m\right):$ (a) $\Delta \lambda_{\text {em }}=$ $60 \mathrm{~nm}$; (b) $\Delta \lambda_{\mathrm{em}}$ varied.

obtained drops rapidly with the decrease of $R_{1}$ and the increase of $A_{2}$. Roughly, with $R_{1}<0.5$ or $A_{2}>0.25$, one no longer observes enhancement (i.e. $G_{\text {int }}<1$ ). In the ideal (lossless) case of $A_{2}=0, G_{\text {int }}$ as high as $\sim 4$ may be obtained.

Fig. 6(b) shows calculated $G_{\text {int }}$ as a function of $R_{2}$ with $R_{1}=0.9, A_{2}=0.10$, and $\Delta \lambda_{\mathrm{em}}=30-100 \mathrm{~nm}$. Narrower molecular emission spectra better match the peak region of the cavity mode [Fig. 5(a)], and thus give substantially larger enhancement with the same cavity structure. In Fig. 6, of particular interest is the value of $R_{2}$ for achieving maximal $G_{\text {int }}$ in each curve. It can be quickly estimated with $R_{2}=R_{1}\left(1-A_{2}\right)^{2}$, which maximizes $G_{\mathrm{cav}}\left(\lambda_{\mathrm{em}}\right) / G_{\mathrm{con}}\left(\lambda_{\mathrm{em}}\right)$ and is obtained by solving $d\left[G_{\text {cav }}\left(\lambda_{\text {em }}\right) / G_{\text {con }}\left(\lambda_{\text {em }}\right)\right] / d \sqrt{ } R_{2} \quad \approx d\left[\left(1-R_{2}-\right.\right.$ $\left.\left.A_{2}\right) /\left(1-\sqrt{ } R_{1} \sqrt{ } R_{2}\right)^{2}\right] / d \sqrt{ } R_{2}=0$. True optimal $R_{2}$ is slightly lower than this value, so that FWHM of $G_{\text {cav }}(\lambda)$ expands slightly to better match $\Delta \lambda_{\mathrm{em}}$ (meanwhile the peak value of $G_{\text {cav }}(\lambda)$ also reduces).

\section{B. Rigorous Electromagnetic Modeling}

The Fabry-Perot formulation like (1) treats only the optical characteristics along the forward direction of an OLED, in which there is no difference for $s$ or $p$ polarizations. However, when considering detailed emission characteristics along different viewing angles of an OLED, the dependence on both wavelength and polarization must be considered. Furthermore, in the more accurate treatment of optical characteristics of OLEDs, the influence of cavity on the transition rate of molecular excited states must be included. All these can be considered by adopting the rigorous and full-vectorial electromagnetic modeling of OLEDs.

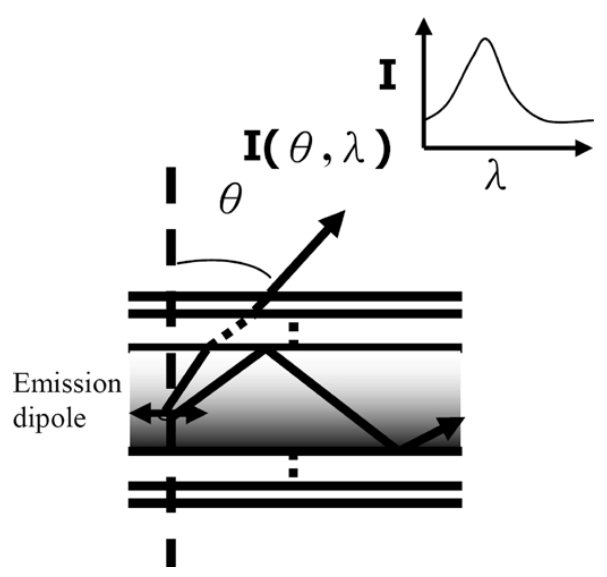

Fig. 7. Illustration of radiation from an oscillating dipole of a particular position, orientation, and frequency embedded in a general layered structure.

Such a rigorous electromagnetic model adopts a classical approach based on the equivalence between the emission of a photon due to an electrical dipole transition and the radiation from a classical electrical dipole antenna, which can take into account losses due to electrodes and absorption [19], [28]-[30]. The formulations are rather complicated, and therefore, in the following only brief outlines of the treatment are given. First, consider the radiation field from a single dipole of particular position, orientation and frequency embedded in a general layered structure, as illustrated in Fig. 7. With plane-wave expansion of the dipole field, the full-vectorial electromagnetic fields generated by a radiation dipole embedded in a layered structure is calculated, from which the distribution of the radiation power into different plane-wave modes and the far-field radiation related to emission characteristics of an OLED are obtained [19], [28]-[30]. In the plane-wave expansion, each plane-wave mode can be characterized by an in-plane wave vector $k_{t}$, where $k_{t}$ is the component of the wavevector parallel to the layer surface. To model emission characteristics of a real OLED, one treats the emitting layer as an ensemble of mutually incoherent dipole radiators with distributions in dipole orientations, locations, and frequencies (using the photoluminescence (PL) spectrum of the emitter as the intrinsic spectral distribution of the dipole radiators) [31]. The total emission intensity $I$ from the OLED as a function of the wavelength $\lambda$ and the viewing angle $\theta$ (i.e., $I(\theta, \lambda)$, with $\theta$ measured relative to the surface normal, Fig. 7) is then obtained by averaging contributions over these distributions. Color coordinates of emission at various angles can then be calculated from $I(\theta, \lambda)$.

\section{TOP-EMITTING OLEDS}

Conventional OLEDs (Fig. 1), adopting reflective top metal cathodes, transparent bottom anodes and transparent substrates, are bottom-emitting devices which emit light from their bottom surface (substrate). In AMOLED displays, however, the pixel circuits of opaque thin-film transistors (TFTs) below OLEDs block emission from a significant portion of the pixel area, reducing the aperture ratio of pixels [Fig. 8(a)]. OLED displays with lower aperture ratios would require higher OLED current densities to achieve desired display brightness, detrimental to the efficiency and lifetime of OLED operation. Top-emitting 
(a)
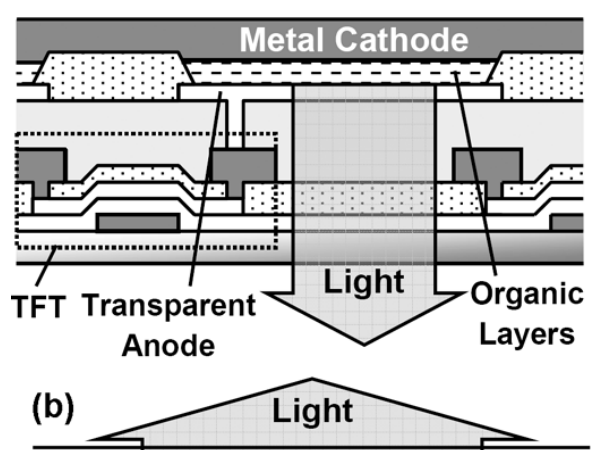

Transparent Cathode

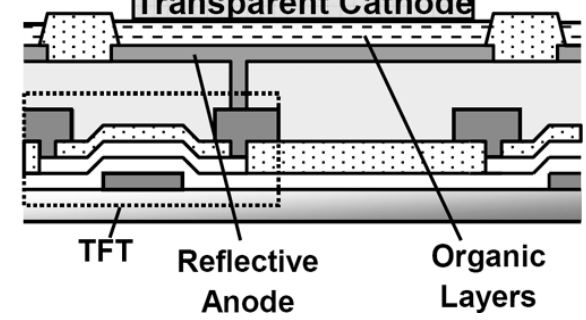

Fig. 8. Schematic diagrams showing the cross sections of AMOLED displays with: (a) top-emitting and (b) bottom-emitting configurations.

OLEDs, i.e., OLEDs that emit light from the top surface of devices remove such constraints, and therefore render feasible fabrication of OLED displays on opaque substrates such as $\mathrm{Si}$ wafers and also permit use of more complicated pixel circuits in AMOLEDs for higher display quality yet without sacrificing aperture ratios of pixels [Fig. 8(b)] [13], [14]. Top-emitting OLEDs, in general, are composed of a reflective bottom anode, organic layers, and a (semi-)transparent top cathode for light out-coupling [Fig. 8(b)]. The major challenges for making efficient top-emitting OLEDs are to develop (semi-)transparent top cathodes with effective electron injection and to develop the reflective bottom anode with efficient hole injection, since they are different from the well-developed electrode structures in conventional OLEDs. Hence, development of these electrode structures with desired electrical and optical properties has been the major interest of investigation.

\section{A. Development Of (Semi-)Transparent Top Cathodes}

A straightforward way of preparing a transparent top cathode is to deposit a transparent conductor, usually sputtered ITO, on top of OLEDs. Yet, ITO in general is not an appropriate electron-injection electrode for typical organic electron-transport materials and also sputtering of ITO could easily damage organic layers beneath, which were difficulties encountered during the early development of top-emitting OLEDs. In 1996, $\mathrm{Gu}$ et al. first successfully demonstrated transparent top ITO cathodes for OLEDs by room-temperature sputtering [32]. To improve electron injection and to reduce sputtering damage, a thin $(\sim 10 \mathrm{~nm}) \mathrm{Mg}: \mathrm{Ag}$ alloy was deposited between the ITO and the organic electron-transport layer (tris-(8-hydroxyquinoline)aluminum $\left(\mathrm{Alq}_{3}\right)$ as the electron-injection and buffer layer [32] (Fig. 9). A transparency of $\sim 70 \%$ throughout the visible spectral range was obtained for such a cathode structure. In this initial report, since the bottom anode still used ITO, the device actually was a transparent device with emission from both sides

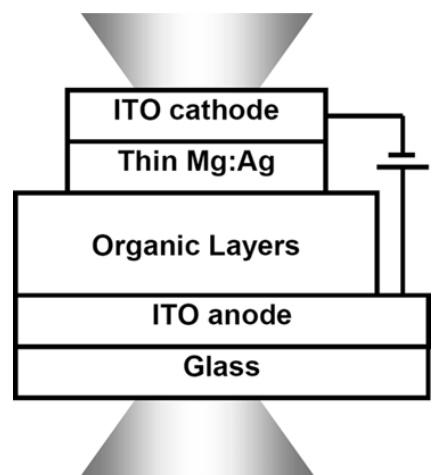

Fig. 9. Device structure of a transparent (double-emitting) OLED using the thin $\mathrm{Mg}$ :Ag metal film as the electron-injection and the buffer layer for the sputtered ITO top cathode.

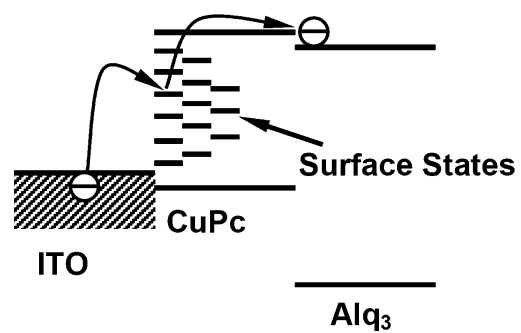

Fig. 10. Illustration of using CuPc as the electron-injection and buffer layer for the sputtered ITO top cathode.

of the device and the total external quantum efficiency achieved was only modest ( $\sim 0.1 \%$ photon/electron) when compared to a control bottom-emitting device $(\sim 1 \%)$. Another critical issue with this first demonstration is that to reduce the sputtering damage, a very low sputtering power of $\sim 5 \mathrm{~W}$ was used for the ITO deposition, resulting in a very slow (and thus not practical) deposition rate of $0.05 \AA / s$. After Gu's initial work, a few other low-work-function metals (e.g. Ca, Al etc.) had also been tested as the electron-injection and buffer layer, the ITO deposition rate had been significantly raised (up to $1.5 \AA / \mathrm{s}$ ) using higher sputtering power and more optimized condictions, and device performances had been largely improved to the level of conventional OLEDs [33], [34].

Shortly afterwards, Parthasarathy et al. found that some more robust organic materials, such as copper phthalocyanine $(\mathrm{CuPc}$, 3-6 nm), can also serve as the electron-injection and the buffer layer for ITO, and demonstrated metal-free transparent top cathodes and devices [35]. It was suggested that in such a device, the formation of surface states at the CuPc/ITO interface facilitates electron injection into the device (Fig. 10). Compared to OLEDs with the Mg:Ag/ITO cathode, such devices exhibit higher transparencies ( $\sim 85 \%$ at the peak) and a higher quantum efficiency ( $\sim 0.4 \%)$.

The transparent cathode based on $\mathrm{Alq}_{3}-\mathrm{CuPc}-\mathrm{ITO}$ was further improved by inserting an ultrathin $\mathrm{Li}$ layer $(<1 \mathrm{~nm})$ prior to ITO deposition to enhance electron injection at $\mathrm{CuPc} / \mathrm{ITO}$ and $\mathrm{Alq}_{3}-\mathrm{CuPc}$ interfaces, as reported by Hung and Tang [36]. Transparent OLEDs with the CuPc-Li-ITO cathode exhibited current-voltage characteristics and efficiency similar to those of control devices with the conventional Mg:Ag cathode. In addition, the ITO deposition rate was raised up to a more practical 


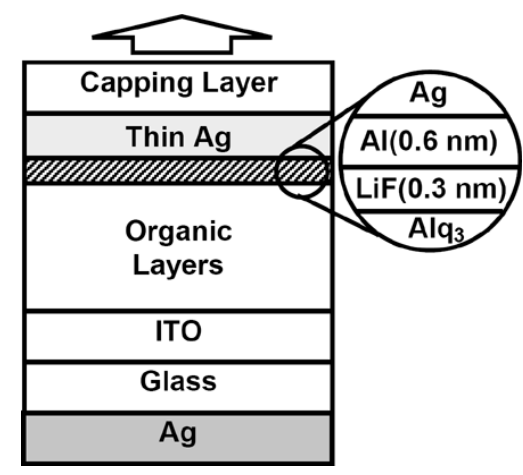

Fig. 11. Top-emitting OLED using a semitransparent thin metal layer capped with a transparent dielectric layer as the semitransparent top cathode.

value of $10 \mathrm{~nm} / \mathrm{min}$. By replacing CuPc with 2,9-dimethyl-4,7 diphenyl-1,10-phenanthroline (BCP), Parthasarathy et al. further showed that placing $\mathrm{Li}$ on either side of the BCP layer in the $\mathrm{Alq}_{3}-\mathrm{BCP}-\mathrm{ITO}$ cathode structure improves device characteristics and concluded that the effect of the $\mathrm{Li}$ layer is associated with the diffusion of $\mathrm{Li}$ atoms into the buffer layer [37]. Very recently, Kanno et al. demonstrated highly efficient (10\% external quantum efficiency) top-emitting phosphorescent white OLEDs using the fully transparent top ITO cathode and using an electron-transport layer with n-type conductive doping (Li doped diphenyl-1,10-phenanthroline, i.e., BPhen:Li) as the electron-injection and buffer layer [38].

Yamamori et al. introduced transparent metal acethylacetonate complexes, such as $\mathrm{Ni}(\mathrm{acac})_{2}, \mathrm{Mg}(\mathrm{acac})_{2}$, and $\mathrm{Ca}(\mathrm{acac})_{2}$, as the new electron-injection and buffer materials for the transparent top electrode [39]. Besides, ITO had been replaced by zinc-doped indium oxide (IDIXO) as the transparent top electrode in this work, which could form amorphous films of low resistance with room-temperature sputtering. Furthermore, Yamamori et al. introduced intermittent low-power sputtering followed by continuous high-power sputtering as a means to reduce sputtering damage and to increase the deposition rate. The device showed very high transparency of $>80 \%$ from 450 to $750 \mathrm{~nm}$.

In general, the top cathodes based on sputtered transparent conductors has complications in sputtering-induced damage and the capability of electron injection, which would lead to degraded device performances or slow deposition conditions and thus make them less compatible with conventional OLED fabrication. In view of these, Hung et al. introduced the semitransparent top cathode based on thin metal layers that could be simply fabricated by thermal evaporation and are more compatible with conventional OLED fabrication [40]. The semitransparent cathode consisted of the ultrathin $\mathrm{LiF}(0.3$ $\mathrm{nm})-\mathrm{Al}(0.6 \mathrm{~nm})$ bilayer as the electron-injection layer and a thin Ag film (20 nm) as the conductive electrode (Fig. 11), all deposited by thermal evaporation. The device gave electrical characteristics identical to conventional OLEDs. In this work, Hung et al. also introduced the new concept of using a dielectric capping layer of high refractive index (preferably also deposited by thermal evaporation) as the index-matching layer for enhancing optical transmission of the semitransparent metal cathode (Fig. 11). In general, any transparent materials, either dielectric, semiconductive, or conductive, with high enough refractive indices could be used as the capping layer, yet the semiconductive or conductive capping layer could contribute to electrical conduction as well. Using the device structure of Ag-coated glass substrate/ITO/arylamine/Alq ${ }_{3}-\mathrm{LiF} \quad(0.3$ $\mathrm{nm})-\mathrm{Al}(0.6 \mathrm{~nm})-\mathrm{Ag}(20 \mathrm{~nm}) / \mathrm{Alq}_{3}$ (as the dielectric capping layer) (Fig. 11), Hung et al. showed that the device can achieve a $~ 90 \%$ efficiency of a conventional bottom-emitting OLED.

Lu et al. [41] demonstrated truly top-emitting phosphorescent OLEDs (instead of transparent or double-emitting devices) using ITO-overcoated $\mathrm{Ag}$ and $\mathrm{Ca}(20 \mathrm{~nm})-\mathrm{ITO}(80 \mathrm{~nm})$ as the highly reflective bottom anode and the (semi-)transparent top cathode, respectively. The thin ITO layer on the Ag anode facilitates hole injection into organic layers. The (semi-)transparent cathode may be considered in two ways. In one way, one may regard the thin $\mathrm{Ca}$ metal layer as the electron-injection and buffer layer for the ITO cathode. In the other way, one may regard the high-index ITO $(\sim 1.7-2.1)$ as the index-matching layer for the conductive $\mathrm{Ca}$ electrode. This green phosphorescent OLED exhibited a $15 \%$ higher efficiency $\left(23.1 \mathrm{~cd} / \mathrm{A}\right.$ at $\left.10 \mathrm{~mA} / \mathrm{cm}^{2}\right)$ than the control bottom-emitting OLED (20.3 cd/A). Lu et al. argued that the slight efficiency enhancement with the top-emitting device was due to elimination of optical trapping in the substrate.

Later, Riel et al. introduced a wide-gap semiconductor $\mathrm{ZnSe}$ that could be thermally evaporated and has very high refractive index of $\sim 2.6$, as the capping layer for the (semi-)transparent metal cathode $(12 \mathrm{~nm} \mathrm{Ca} / 12 \mathrm{~nm} \mathrm{Mg}$ ) of phosphorescent topemitting OLEDs [20], [42]. The top-emitting OLEDs adopted high-reflection $\mathrm{Al}$ in combination with a thin layer of highwork-function metals such as Pt, Ir, Ni, Pd, or Mo as the hole-injection bottom anode. The authors found that with an optimal ZnSe thickness, a nearly two times enhancement in the cd/A efficiency in comparison with that of top-emitting devices using no capping layer (64 cd/A versus $38 \mathrm{~cd} / \mathrm{A}$ ) was obtained. Surprisingly, the authors found that the optimal capping thickness is not that giving largest transmission, and instead it is closer to that giving lowest transmission. Such results are contradictory to the concept proposed by Hung et al. [40], but no clear explanation was provided. Similarly, Chen et al. and Hsu et al. later reported the semitransparent cathode structures of Ag (20-25 $\mathrm{nm}) / \mathrm{TeO}_{2}(n \sim 2.3-2.5)$ and $\mathrm{Ca}(5 \mathrm{~nm})-\mathrm{Ag}(15 \mathrm{~nm}) / \mathrm{SnO}_{2}$, respectively, for various top-emitting OLEDs, top-emitting microcavity OLEDs, and top-emitting white OLEDs, etc. [27], [31], [43], [44]

To understand the mechanism of enhancement in cd/A efficiencies of top-emitting OLEDs using the combination of semitransparent metal/capping layer as the top cathode, recently Lin et al. performed detailed theoretical and experimental studies [27]. In their work, Lin et al. used ultrathin LiF/Al as electron-injection layer, a thin $\mathrm{Ag}$ film as the conductive electrode, and high-index $\mathrm{TeO}_{2}(n \sim 2.3-2.5)$ as the capping layer. Similar to $\mathrm{ZnSe}, \mathrm{TeO}_{2}$ also can be deposited by thermal evaporation and has high transparency. According to Lin's studies, top-emitting OLEDs using metal electrodes for both bottom and top electrodes indeed form rather strong optical microcavities. As discussed in Section II-A, it is possible to obtain substantial enhancement in cd/A efficiency (in the forward direction) relative to conventional noncavity bottom-emitting devices if elec- 


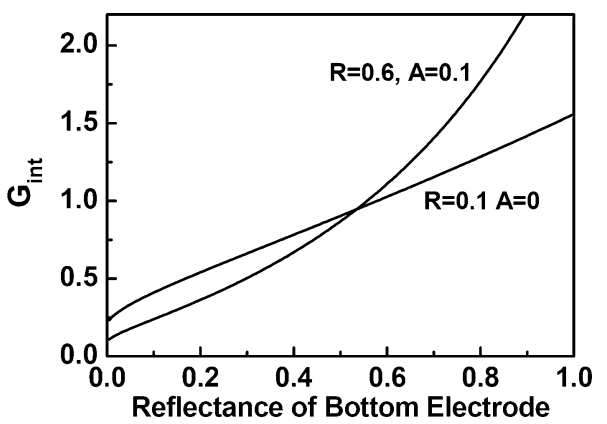

Fig. 12. Calculated $\mathrm{G}_{\text {int }}$ of the top-emitting OLED as a function of the reflectance of the bottom electrode using: (a) a fully transparent top electrode (transmittance $T=0.9$, reflectance $R=0.1$, absorptance $A=0$ ) and (b) a high-reflection low-loss top electrode (transmittance $T=0.4$, reflectance $R=0.6$, absorptance $A=0.1$ ).

trode mirrors are of high enough reflectivity and low enough loss. Therefore, in such cases, the efficiency enhancement is due to strong microcavity effects rather than simply due to enhanced transmission as Hung et al. originally suggested [40]. In such a view point, top-emitting OLEDs incorporating semitransparent metal top electrode, if optimized according to microcavity conditions, would naturally give larger cd/A efficiencies than top-emitting OLEDs using fully transparent top electrode, since the microcavity effect in the latter is weak. As such, top-emitting OLEDs using fully transparent top electrodes, even optimized, would give efficiency only similar to that of conventional noncavity bottom-emitting devices, since both are weakmicrocavity devices. The strong microcavity effects and devices will further discussed in Section VI (microcavity OLEDs).

\section{B. Development of Reflective Bottom Anodes}

Using the method provided in Section II-A and the same assumption in emitters, one may evaluate $G_{i n t}$ of an optimized top-emitting OLED as a function of the reflectivity of the bottom anode. $\mathrm{G}_{\text {int }}$ for two cases of using either a fully transparent top cathode (with a transmittance of 0.9 ) or a high-reflection low-loss top cathode (with a reflectance of 0.6 and an absorptance of 0.1) are shown in Fig. 12. Clearly, a high-reflectivity bottom anode is critical for obtaining high efficiency in either case. Among various metals, Ag and Al have the highest reflectivity over the visible wavelengths. $\mathrm{Ag}$ and $\mathrm{Al}$, however, in general are not considered as ideal hole-injecting anode for OLEDs due to their rather low work function ( $4.1-4.5 \mathrm{eV})$, not matching well the ionization potentials of organic materials commonly used in OLEDs. On the other hand, metals that have high work functions better matching the ionization potentials of general organic hole-transport materials, such as $\mathrm{Pt}, \mathrm{Ir}, \mathrm{Ni}, \mathrm{Pd}$, and Mo, etc., have rather low reflectivity [45].

In view of these, effective bottom anodes for top-emitting OLEDs in general use high-reflectivity $\mathrm{Ag}$ or $\mathrm{Al}$ overcoated with a thin hole-injection layer to achieve both desired optical and electrical properties. Such a hole-injection layer could be a high-work-function metal such as $\mathrm{Pt}, \mathrm{Ir}, \mathrm{Ni}, \mathrm{Pd}$, and $\mathrm{Mo}$, etc., or the traditional anode conductor ITO [20], [38], [41], [42], as in a few cases of Section III-A. Beierlein et al. and other groups also reported that some metal oxides having high work functions or large ionization potentials, such $\mathrm{NiO}_{x}, \mathrm{~V}_{2} \mathrm{O}_{5}$, and

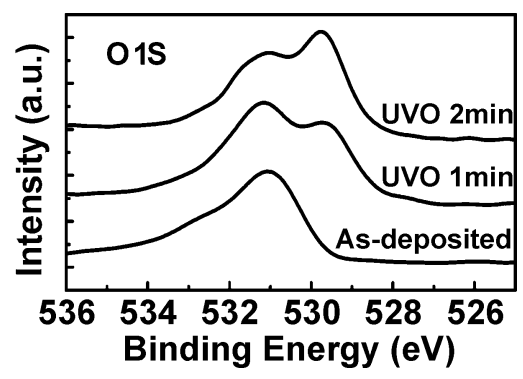

Fig. 13. Core-level O 1s XPS spectra of an as-deposited Ag film and a UV-ozone-treated Ag film.

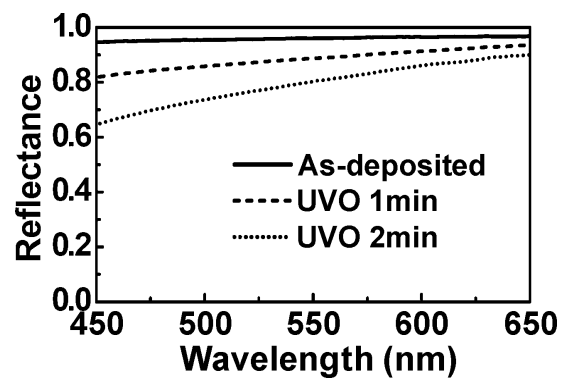

Fig. 14. Reflection spectra of an as-deposited Ag film and a UV-ozone-treated (UVO) Ag film.

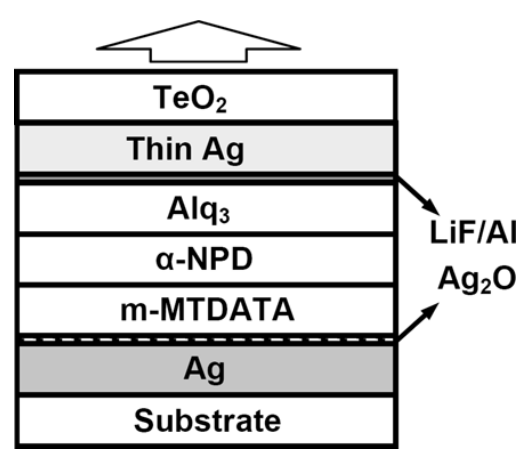

Fig. 15. The device structures of the top-emitting device using the UV-ozone-treated $\mathrm{Ag}$ as the bottom anode and thin $\mathrm{Ag}$ capped with $\mathrm{TeO}_{2}$ as the semi-transparent top cathode.

$\mathrm{Pr}_{2} \mathrm{O}_{3}$ etc. [38], [46], [47], can also be used as the hole-injection layer for $\mathrm{Al}$ and $\mathrm{Ag}$. These metal-oxide layers could be deposited by different methods, including thermal evaporation, sputtering, and chemical vapor deposition [38], [46], [47]. Alternatively, they may be formed by oxidizing the corresponding metals with ozone or oxygen plasma [38], [43]. As an example, Chen et al. reported that the hole injection from a Ag bottom anode into organic layers could be substantially enhanced by inducing a thin layer $(<100 \AA)$ of $\mathrm{AgO}_{x}$ on the surface of $\mathrm{Ag}$ with the UV ozone treatment [43]. The formation of $\mathrm{AgO}_{x}$ was confirmed by XPS measurements (Fig. 13) and such UV-ozone treated $\mathrm{Ag}$ anode still retained a high reflectance of $82 \%-91 \%$ over the visible range, only slightly lower than that of an as-deposited Ag film (Fig. 14). Such an anode gives electrical characteristics competitive with those of the conventional ITO anode. The top-emitting devices using this $\mathrm{Ag} / \mathrm{AgO}_{x}$ anode and the thin $\mathrm{Ag} / \mathrm{TeO}_{2}$ top cathode (Fig. 15) exhibited rather strong microcavity effects and an enhancement in the cd/A efficiency compared to those of a noncavity bottom-emitting device. 


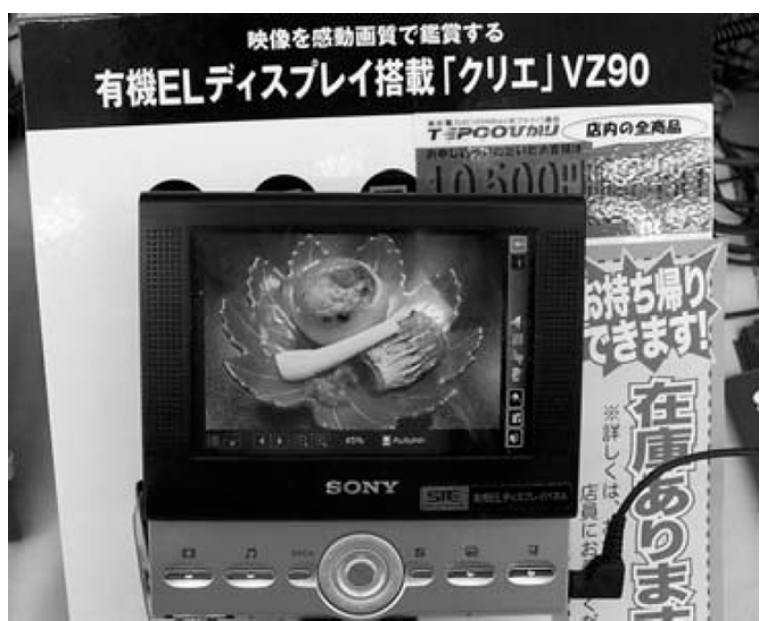

Fig. 16. SONY's 3.8-in full-color top-emitting AMOLED displays for PDA products (CLIé PEG VZ-90, commercialized in 2004).

Two other different approaches have also been reported as effective hole-injection schemes for the reflective bottom anode. First, an organic hole-transport layer with p-type conductive doping may be used for this purpose, since with such a p-type conductive organic layer, the choice of the bottom anode and its work function is much less critical [27]. In addition, Xie et al. and Hsu et al. demonstrated that depositing an ultrathin $(\sim 0.3$ $\mathrm{nm}$ ) plasma-polymerized hydrocarbon film $\left(\mathrm{CF}_{x}\right)$ at the surface of the Ag anode can also significantly enhance the hole-injection properties [44], [48].

\section{Applications in AMOLED Displays}

Aiming at OLED microdisplays on Si chips, eMagin Corporation was the first to incorporate top-emitting OLEDs into AMOLEDs [13], while in 2001 Sony Corporation was the first to demonstrate large-area top-emitting AMOLEDs on glass substrates [14]. Sony's 13.1-in full-color SVGA $(800 \times 600)$ top-emitting AMOLED prototype was the ever largest OLED display at that time. In 2004, Sony Corporation launched its first top-emitting AMOLED product (Fig. 16), which is a 3.8-in HVGA $(480 \times 320)$ full-color AMOLED for PDA applications. By employing top-emitting OLED structures and corresponding microcavity effects, such OLED displays exhibit higher brightness $\left(150 \mathrm{~cd} / \mathrm{m}^{2}\right.$ versus $\left.55 \mathrm{~cd} / \mathrm{m}^{2}\right)$, higher contrast ratio (1000:1 versus 100:1), and larger color gamut ( $\sim 100 \%$ versus $40 \%$ NTSC), compared to Sony's equal-level LCD displays.

By utilizing OLEDs having a transparent ITO bottom anode and a semitransparent top cathode, in 2003 International Symposium of Society of Information Display (SID 2003), Samsung Display Inc. (SDI) demonstrated a 2.2-in transparent AMOLED that could display image on both sides (Fig. 17) [49]. An aperture ratio of $32 \%$ and a transparency of $20 \%$ were achieved. Due to the lower transparency of the top cathode, the top side gave only half of the brightness of the bottom side. In SID 2004, Nakamura et al. demonstrated a 2.1-in dual-side emission AMOLED [50] with improved aperture ratio (41\%), improved transparency (30\%), and equal brightnesses on both

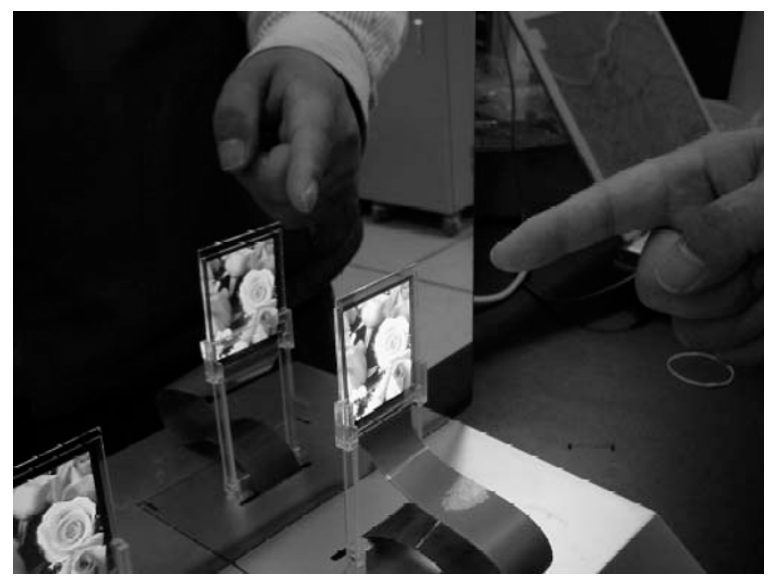

Fig. 17. The 2.2-in full-color transparent (double-emitting) AMOLED demonstrated by Samsung SDI Corporation in SID 2003.

(a)

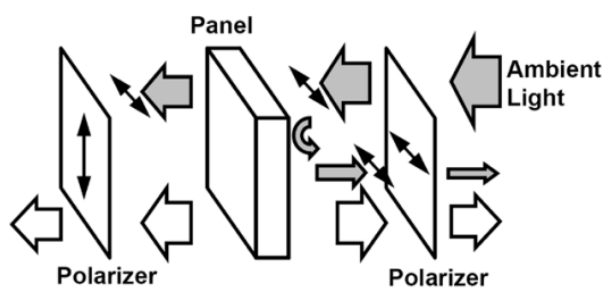

(b)

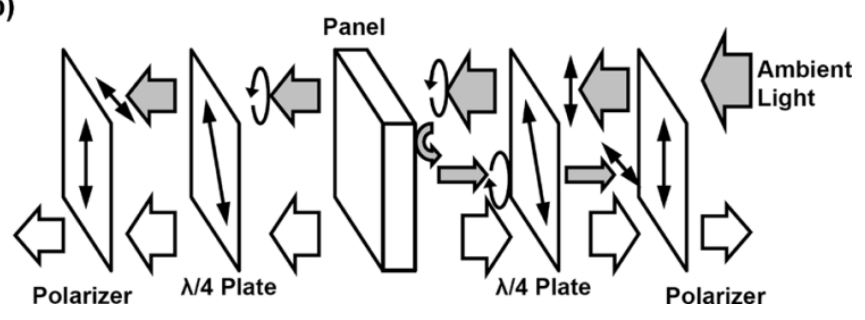

Fig. 18. High-contrast double-emitting OLED displays: (a) using combinations of linear polarizers and (b) using combinations of linear polarizers and quarter-wave plates.

sides. In general, transparent displays would suffer a low contrast ratio due to transmission of ambient light. By laminating linear polarizers and quarter-wave plates on both sides of the display to eliminate transmission and reflection of ambient illumination (Fig. 18), Nakamura et al. further demonstrated non-transparent dual-emission AMOLED with high contrast ratios $(>400)$. Such dual-emission displays may find uses in portable electronics (e.g., mobile phones) requiring image displaying on both sides.

In SID 2005, by combining both bottom-emitting and top-emitting OLEDs in a single pixel and using independent TFT pixel circuits for each (Fig. 19), AU Optronics Corporation demonstrated a 1.5-in double-emitting AMOLED which can independently display two different images on both sides of the display panel [51]. Based on a different idea of fabricating a single OLED having a top-emitting portion on top of the opaque TFT area of a pixel and having a transparent (double-emitting) portion in the remaining area (Fig. 20), Universal Display Corporation also demonstrated an AMOLED display that can display images (not independently, however) on both sides of the panel [52]. With such a configuration, equal brightnesses on 


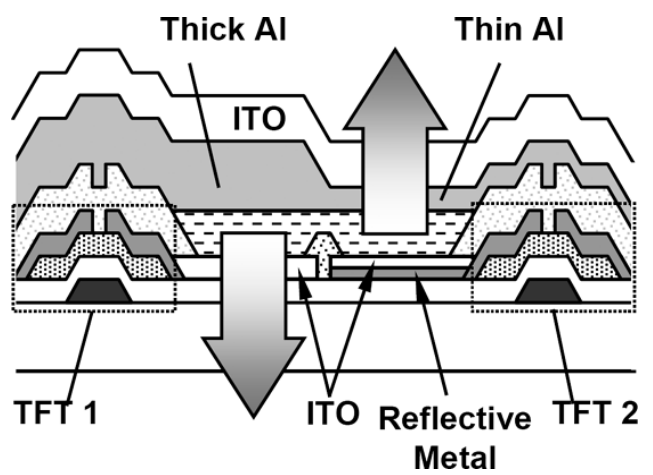

Fig. 19. Schematic diagram showing the cross section of the double-emitting AMOLED reported by AU Optronics Corporation in SID 2005. The display combines independently controlled top-emitting and bottom-emitting OLEDs in a single pixel and can independently display two different images on both sides of the display panel.

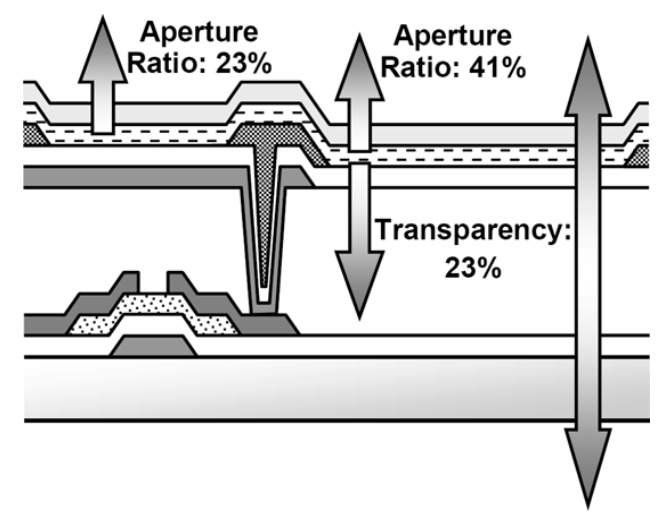

Fig. 20. Schematic diagram showing the cross section of the double-emitting AMOLED reported by Universal Display Corporation in SID 2005. The display contains pixel OLEDs having a top-emitting portion on top of the TFT area and having a transparent (double-emitting) portion in the other area. (a)

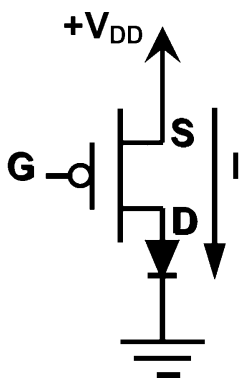

(b)

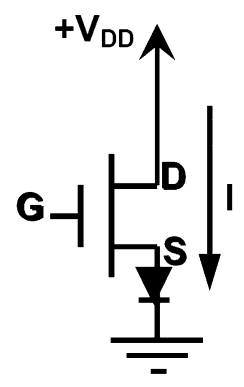

Fig. 21. Portion of the AMOLED pixel circuit that contains the driving transistor in series with a conventional pixel OLED having the anode at bottom. (a) Driving transistor is a p-type transistor. (b) Driving transistor is a n-type transistor.

both sides could be achieved, even though there exist reflection and absorption in the semitransparent top cathode.

\section{INVERTED OLEDS}

With the advance of the AMOLED technologies, a variety of pixel circuits have been developed for improving the display performance. Whatever the detailed pixel circuit is like, always there is at least one transistor (i.e., the driving transistor) in series with the pixel OLED (Fig. 21). The remaining part of the pixel circuit controls the gate voltage of the driving transistor, (a)

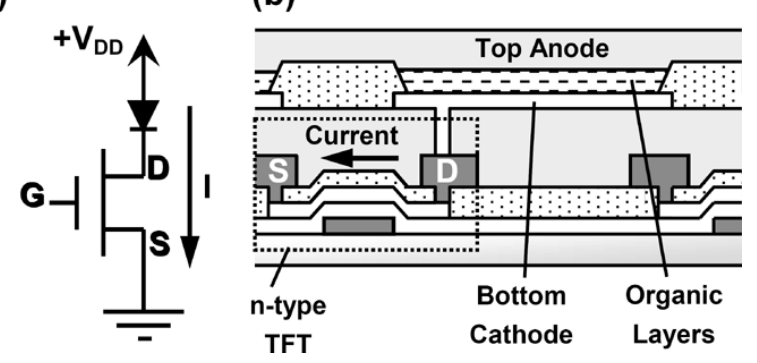

Fig. 22. (a) Portion of the AMOLED pixel circuit that contains the n-type driving transistor in series with an "inverted" pixel OLED having the cathode at bottom. (b) Cross section of the AMOLED using a n-type transistor to drive the inverted OLED.

which in turn controls the current through the transistor and the OLED and consequently, the OLED brightness. Since the conventional OLED has the anode at bottom and the cathode on top, it is electrically connected to the driving transistor through the anode, as shown in Fig. 8. When the driving transistor is $\mathrm{p}$ type, one obtains the partial circuit of Fig. 21(a), in which the OLED anode is connected to the drain of the transistor. When the transistor is $n$ type, one obtains the circuit of Fig. 21(b), in which the OLED anode is connected to the source of the transistor. The circuit of Fig. 21(a) operates like a voltage-controlled current source (VCCS) to OLED, since the transistor current supplied to OLED is controlled by the gate voltage independent on OLED operation (if the transistor is operated in the saturation mode). Fig. 21(b), however, operates like a source follower, since the OLED voltage follows the source voltage of the transistor. In such a configuration, the gate voltage is a sum of the transistor gate-to-source voltage $\left(\mathrm{V}_{\mathrm{GS}}\right)$ and the OLED voltage, and the transistor current (OLED current) is not independently controlled by the gate voltage any more. Usually, the VCCS configuration is preferred to the source-follower configuration, since in the latter case the variation or degradation of either device will simultaneously affect the operation of the other, making it more difficult to fix with circuit designs. However, as shown in Fig. 8, with the conventional OLED structure, it is only possible to form the VCCS configuration with the p-type transistor. Indeed there is a strong desire to form the VCCS configuration with the n-type transistor, since usually the n-type transistors have performances significantly superior to those of p-type transistors. For instance, in amorphous $\mathrm{Si}(\mathrm{a}-\mathrm{Si})$ TFTs often used in large-scale AMLCDs and AMOLEDs, only n-type transistors are available, while in polycrystalline silicon (poly-Si) TFTs, n-type TFTs usually have higher carrier mobility and thus lower operation voltage. To form the VCCS configuration with the n-type transistor [see Fig. 22(a)], one must "invert" the OLEDs, i.e. making OLEDs that have a cathode at bottom and an anode on top, as shown in Fig. 22(b).

The major challenges in inverted OLEDs, like in developing top-emitting OLEDs, are to prepare a bottom cathode providing effective electron injection and to prepare a top anode providing effective hole injection. Baigent et al. reported the first inverted (top-emitting) OLED with polymer OLEDs in 1994 [53]. In this early work, an Al layer and a sputtered ITO were employed as the bottom cathode and the top anode, respectively, for the 

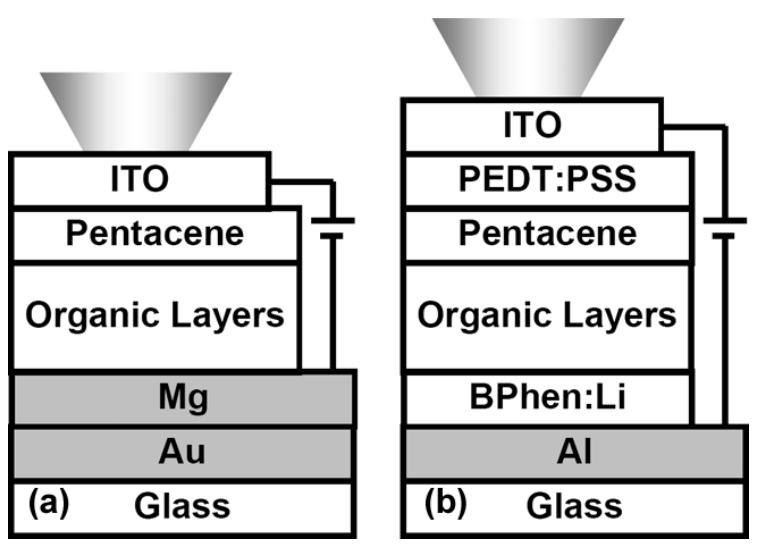

Fig. 23. Two structures of inverted top-emitting OLEDs having: (a) $\mathrm{Mg}$ as the bottom cathode and having the sputtered ITO top anode with Pentacene as the hole-injection buffer layer and (b) Al bottom cathode with BPhen:Li as the electron-injection layer and having the sputtered ITO top anode with Pentacene/PEDT:PSS as the hole-injection buffer layer.

single-layer $\operatorname{poly}(p$-phenylene vinylene) (PPV) device fabricated on Si substrates. The device performances, however, were insufficient for real applications.

1997, Bulovic et al. demonstrated an inverted top-emitting OLED for small-molecule devices using a low-work-function $\mathrm{Mg}: \mathrm{Ag}$ alloy as the bottom cathode and an sputtered ITO as the top anode [54]. As in the case of fabricating an ITO top electrode for top-emitting OLEDs described in Section III, a more robust organic layer based on 3, 4, 9, 10-perylenetetracarboxylic dianhydride (PTCDA) or CuPc was employed as the buffer layer for reducing the sputtering-induced damage. Relatively low device efficiencies $(0.15 \%-0.3 \%)$ in comparison with conventional OLEDs indicate there were still issues in carrier injection from electrodes. Following Bulovic's work, Dobbertin et al. later introduced the pentacene, a typical organic TFT material with high hole mobilities, as the buffer-hole-injection layer for the sputtered top ITO anode [55]. The inverted top-emitting OLED [Fig. 23(a)] adopted the two-layer bottom cathode of $\mathrm{Au}(100 \mathrm{~nm}) / \mathrm{Mg}(100 \mathrm{~nm})$. It showed that a thicker pentacene layer gave better protection against sputtering, yet also resulted in lower device efficiency due to rather strong absorption in pentacene. With an optimal pentacene thickness $(43 \mathrm{~nm})$, the device exhibited an efficiency of $3.9 \mathrm{~cd} / \mathrm{A}$ with nondoped $\mathrm{Alq}_{3}$, which is a significant improvement over previous results. Shortly afterward, Dobbertin et al. reported a further improved inverted (top-emitting) OLED with higher cd/A and power efficiencies [Fig. 23(b)] [56]. In the bottom cathode, reactive $\mathrm{Mg}$ was replaced with stable $\mathrm{Al}$ and $\mathrm{Li}$-doped Bphen $(5 \mathrm{~nm})$ was deposited on $\mathrm{Al}$ to improve electron injection into organic layers. In the top anode, a conducting polymer PEDT:PSS (polyethylene dioxythiophene/polystyrene sulfonate) was spin-coated over pentacene to form a composite buffer-hole-injection layer for the top ITO anode.

Very recently, inverted OLEDs have also been demonstrated by Leo et al. with the so-called p-i-n OLED structure [57], [58]. The bottom cathode used transparent ITO. Since ITO in general is not an effective electrode for electron injection into organic materials, an organic electron-transport layer with n-type conductive doping (i.e. Bphen:Li, $15 \mathrm{~nm}$ ) was

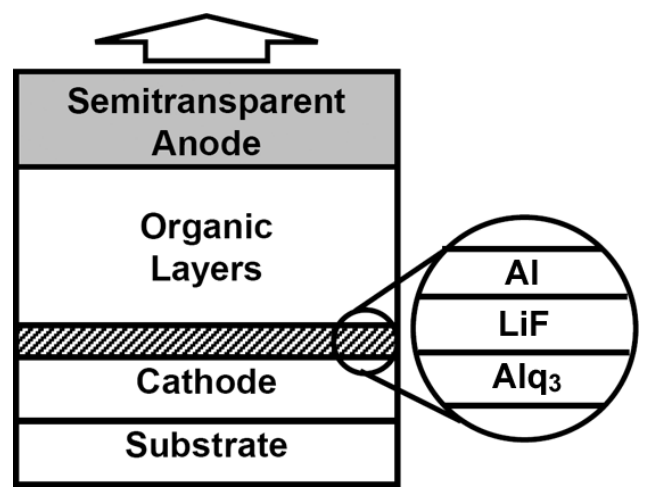

Fig. 24. Device structure of the inverted top-emitting OLED with the ultrathin $\mathrm{Alq}_{3}-\mathrm{LiF}-\mathrm{Al}$ trilayer as the electron-injection layer.

deposited on top of ITO to facilitate electron-injection and conduction. The top anode adopted the thin $\mathrm{Au}(10 \mathrm{~nm})$ or ITO as the (semi-)transparent electrode, along with an organic hole-transport layer (4, 4', 4"-tris(3-methylphenylphenylamino)triphenylamine, m-MTDATA) with p-type conductive doping (tetrafluorotetracyano-quinodimethane, $\mathrm{F}_{4}$-TCNQ) below to facilitate hole-injection and conduction. The devices thus were transparent inverted OLEDs. These devices achieved practical brightness of $100 \mathrm{~cd} / \mathrm{m}^{2}$ at voltages below $4 \mathrm{~V}$, a usual feature of $\mathrm{p}$-i-n OLEDs.

In all the inverted OLEDs mentioned above, the preparation of electron-injecting bottom cathodes all involves handling highly reactive low-work-function metals, such as $\mathrm{Mg}$ and $\mathrm{Li}$, during fabrication. In one way, they were deposited as the cathode layer directly. However, changing the formation process of the contact leads to degraded electron-injecting capability. Furthermore, it is not compatible with real fabrication of AMOLED displays, and thus not practical. In another way, reactive metals were co-deposited with organic electron-transport materials to form the conductive $n$-doped layer for facilitating electron injection from the bottom cathode. Yet, there still remain issues in employing such a technique in production of OLED displays, diffusion of metal dopants, and operational stability. Hence, inverted top-emitting OLEDs involve no handling of reactive metals during fabrication are still of interest.

Very recently, Chen et al. demonstrated efficient inverted top-emitting OLEDs using an ultrathin $\mathrm{Alq}_{3}-\mathrm{LiF}-\mathrm{Al}$ trilayer as the electron-injection structure for the bottom cathode (Fig. 24) [59]. Such an electron-injection structure does not involve handling reactive metals during fabrication and permits use of highly reflective materials such as $\mathrm{Al}$ and $\mathrm{Ag}$ as the bottom cathode. Chen et al. tested the effectiveness of such electron-injection schemes with double-cathode electron-only devices (Fig. 25). The double-cathode electron-only devices have $\mathrm{Ag}$ or $\mathrm{Al}$ with the ultrathin $\mathrm{Alq}_{3}-\mathrm{LiF}-\mathrm{Al}$ trilayer as the bottom cathode and a traditional LiF/Al top cathode. Bottom $\mathrm{Ag}$ or $\mathrm{Al}$ serves as the electron-injecting electrode when biased negatively (reverse bias), and as a counterelectrode for the top cathode when biased positively (forward bias). By comparing current conduction under both forward and reverse biases, one can "benchmark" the electron-injection capability of the bottom cathode with the traditional $\mathrm{LiF}-\mathrm{Al}$ top cathode. As shown 


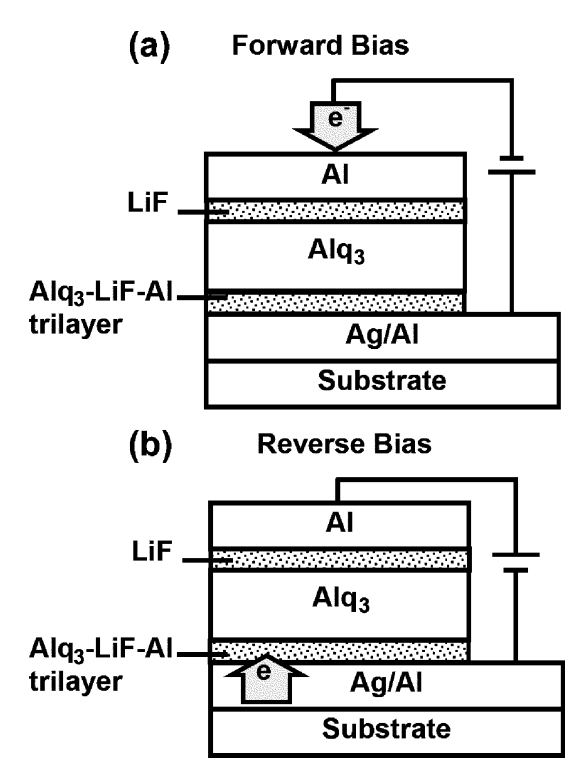

Fig. 25. Double-cathode electron-only device. (a) Under forward bias, electrons inject from the cathode. (b) Under reverse bias, electrons inject from the bottom cathode.
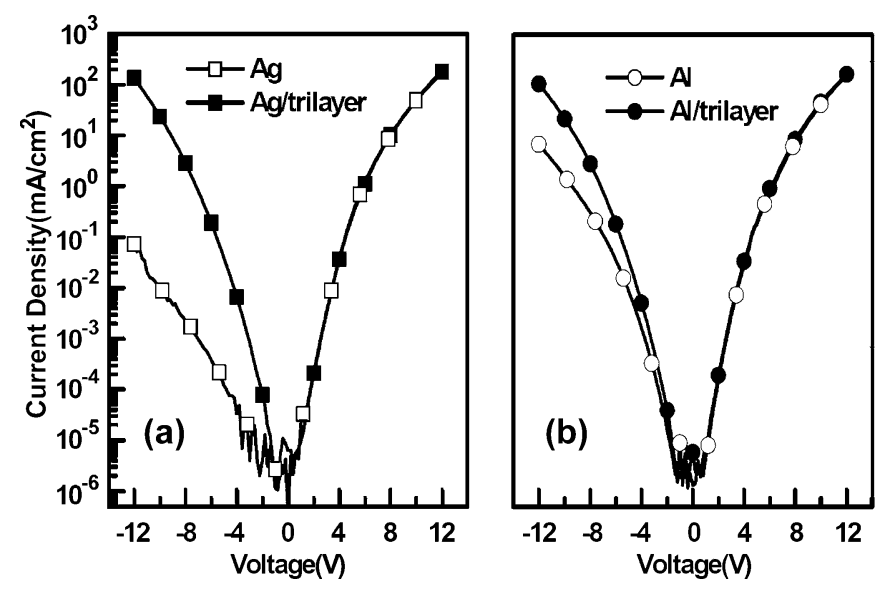

Fig. 26. I-V characteristics of double-cathode devices: (a) using Ag as the bottom cathode with and without the ultrathin $\mathrm{Alq}_{3}-\mathrm{LiF}-\mathrm{Al}$ electron-injection trilayer, and (b) using $\mathrm{Al}$ as the bottom cathode with and without the ultrathin $\mathrm{Alq}_{3}-\mathrm{LiF}-\mathrm{Al}$ trilayer.

in Fig. 26, with the Alq-LiF-Al trilayer, the bottom cathodes show substantially enhancement in electron injection current. Moreover, the electron-injection properties of such bottom cathodes structure are competitive with those of the conventional LiF-Al top cathode. By combining the semitransparent top anode consisting of a m-MTDATA: $\mathrm{F}_{4}$-TCNQ layer as the hole-injection layer, a thin $\mathrm{Ag}$ electrode, and a $\mathrm{TeO}_{2}$ capping layer, Chen et al. accomplished efficient inverted top-emitting OLEDs. The non-doped $\mathrm{Alq}_{3}$ device achieved an efficiency of $5.4 \mathrm{~cd} / \mathrm{A}$, which indeed is higher than that of a conventional bottom-emitting $\mathrm{Alq}_{3}$ device due to microcavity enhancement.

In SID 2005, Murakami et al. reported inverted top-emitting OLEDs employing an e-beam-evaporated $\mathrm{V}_{2} \mathrm{O}_{5}$ layer as a buffer-hole-injection layer for the sputtered IZO (indium zinc oxide) top anode [60]. Such inverted top-emitting OLEDs exhibited identical current-voltage characteristics, comparable operational stability and efficiency in comparison with the

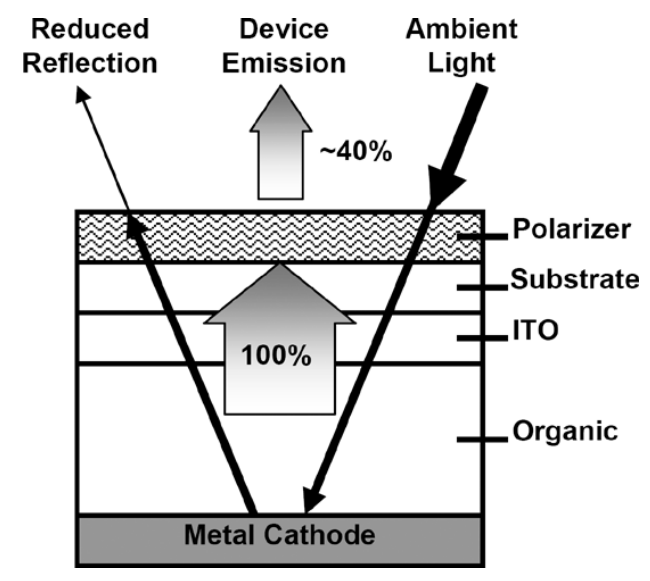

Fig. 27. The schematic illustration of using polarizer films to reduce the reflection and to increase the contrast of an OLED.

conventional bottom-emitting device. In the same conference, Thomson Corporation, Novoled $\mathrm{GmbH}$, and ERSO/IRTI (Electronic Research and Service Organization/Industrial Technology Research Institute) together demonstrated a 3.25-in full-color AMOLED display incorporating inverted top-emitting OLEDs and the poly-Si backplane [61]. The inverted top-emitting OLED adopted the p-i-n structure with $\mathrm{Cr}$ as the reflective bottom electrode and semitransparent metal-dielectric capping as the top anode.

\section{High-CONTRAST OLEDS}

OLEDs, either conventional bottom-emitting or top-emitting, in general are composed of a reflective back electrode, organic layers, and a (semi-)transparent exit electrode for light out-coupling. With the reflective back electrode, OLEDs in general exhibit rather strong reflection. The contrast ratio of a display is defined as the ratio of its brightness at the bright (on) state and its brightness at the dark (off) state. Hence, such reflection would seriously degrade the contrast of an OLED display under a strong lighting environment, since even at the off state, the device will still exhibit some brightness due to reflection of the ambient illumination. Polarizer or filter films may be laminated on the surface of the display panel to reduce reflection of ambient illumination (Fig. 27) [62]. However, the use of such contrast-enhancement films reduces the power efficiency of the displays below $\sim 40 \%$ of the device efficiency due to their strong absorption and also adds extra complexity and cost in fabrication. OLED structures that could integrate the characteristics of low ambient-light reflection within the device structure and yet retain as much emission efficiency will thus be particularly useful, since the display contrast could then be enhanced with reduced impacts on cost and efficiency.

For bottom-emitting OLEDs, several device structures as shown in Fig. 28 have been reported for reducing reflection and for improving contrast. All these structures involve inserting extra layer(s) of optical purposes (absorption, interference or both) into the active region of devices (i.e. between the anode and the cathode). Specifically, these optical layers are inserted neighboring to the reflective top metal cathode. For current conduction, such layers must also have appropriate electrical characteristics (i.e., carrier injection and transport). 
(a)

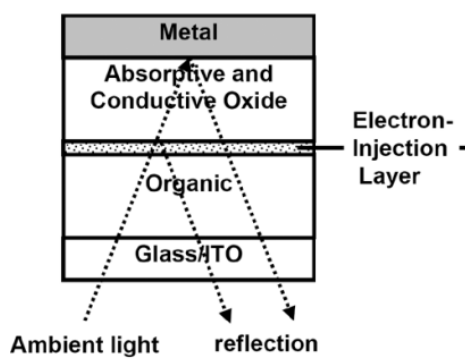

(c)

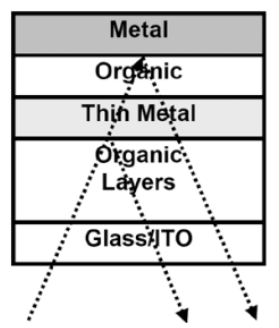

(b)

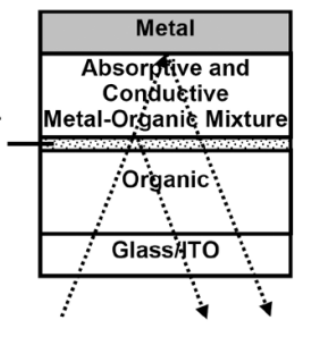

(d)

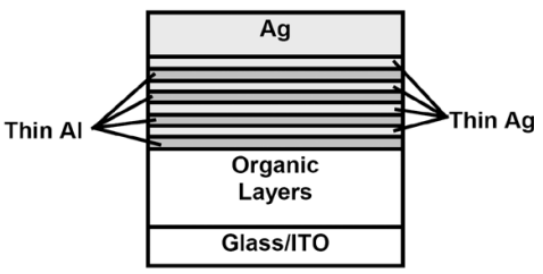

Fig. 28. Several device structures for reducing reflection and improving contrast of bottom-emitting OLEDs: (a) using absorptive and conductive oxide as the "black" layer; (b) using the absorptive and conductive metal-organic mixed layer as the "black" layer; (c) incorporating the metal-organic-metal (MOM) structure; and (d) using the "black" cathode containing alternating nanometer metal-metal layers.

The first type of the high-contrast devices [Fig. 28(a) and (b)] is implemented by inserting an absorptive and conducting layer (the "black" layer) and an ultrathin electron-injection layer (of no optical purposes, e.g. the ultrathin $\mathrm{LiF} / \mathrm{Al}$ bilayer or $\mathrm{Ca}$ ) between the high-reflection metal cathode and the typical organic layers of OLEDs. The reflection of devices is suppressed by both the absorption in the "black" layer and the destructive interference of two most prominent reflections from the reflective cathode and from the organic/"black" layer interface. In 2001, Hung et al. reported the use of thermally evaporated, absorptive and conductive $\mathrm{ZnO}_{x}$ as the "black" layer [see Fig. 28(a)] [62]. Utilizing such a "black" layer in combination with the ultrathin electron-injection layer of $\mathrm{LiF}-\mathrm{Al}$, the reflectance of the device was reduced by nearly one order and the device achieved an efficiency of roughly 50\% of a conventional high-reflection device. On the other hand, Luxell Technologies Inc. and other groups reported the use of thermally co-evaporated $\mathrm{SiO}: \mathrm{Al}$ as the absorptive and conductive oxide layer [63], [64].

Aziz et al. adopted a different approach for the absorptive and conductive "black" layer. Instead of using the conductive oxide, they used the mixture of organic electron-transport materials and metals (e.g., $\mathrm{Alq}_{3}: \mathrm{Ag}, \mathrm{Alq}_{3}: \mathrm{Mg}: \mathrm{Ag}$, etc.) prepared by thermal co-evaporation as the "black" layer [see Fig. 28(b)] [65]. The device with a 150 -nm-thick $\mathrm{Alq}_{3}:$ Ag layer and an electron-injection layer of 2-nm Ca achieved a luminous reflectance of $9.2 \%$ and retained $~ 50 \%$ efficiency of a conventional OLED.

The second type of low-reflection OLEDs [Fig. 28(c)] utilizes mainly the interference mechanism. To reduce reflection, the conventional cathode is replaced with a stack of "thin semitransparent metal-organic electron-transport layer/thick metal" (i.e., the so-called MOM structure). In such a structure, the reflection from the first semitransparent metal layer and that from the second thick metal interfere destructively, leading to reduced

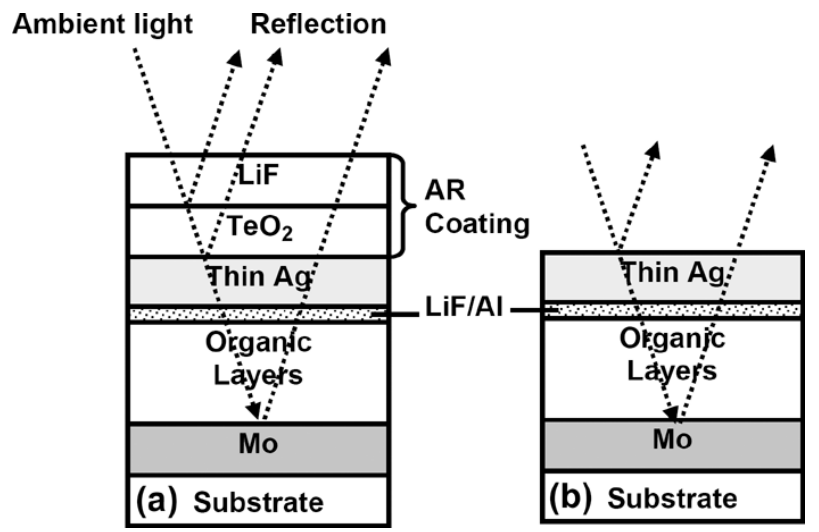

Fig. 29. Low-reflection top-emitting OLEDs: (a) with and (b) without the AR coatings.

reflection. Xie et al. reported the MOM configuration of thin $\mathrm{Sm}-\mathrm{Alq}_{3}$-thick $\mathrm{Al}$ [66], and demonstrated an impressively low luminous reflectance of $\sim 1 \%$. Due to the excellent electron-injection capability of $\mathrm{Sm}$, the device exhibited low operation voltage and an efficiency of slightly $>50 \%$ of a conventional OLED. Similarly, Feng et al. reported the MOM configuration of thin $\mathrm{Al}-\mathrm{Alq}_{3}$-thick $\mathrm{Al}$ for reducing OLED reflection [67].

The third type of low-reflection OLEDs [Fig. 28(d)] utilizes a low-reflection "black" cathode electrode. Li et al. reported the implementation of a "black" cathode with alternating metalmetal layers of nanometers [68]. They demonstrated low-reflection OLEDs using a "black" cathode containing 4 pairs of $\mathrm{Al}$ $(4 \mathrm{~nm})-\mathrm{Ag}(4 \mathrm{~nm})$ layers overcoated with a $28 \mathrm{~nm}$ thick $\mathrm{Ag}$ (Fig. 28(d)). Through characterization with AFM and XPS, Li et al. confirmed that the low reflection was due to formation of aluminum-aluminum oxide nanoclusters within the conducting network of Ag during the slow evaporation process. Such nanostructures contributed to absorption and scattering of the ambient light.

The high-contrast OLED structures described above all deal with bottom-emitting OLEDs. They may not be readily adapted for top-emitting OLEDs that have many technical merits for high-performance AMOLEDs. Recently, Yang et al. reported a high-contrast top-emitting OLED [see Fig. 29(a)][69], [70]. Unlike previous high-contrast devices that all involve inserting extra layer(s) of optical purposes into the active region of devices, Yang's device utilizes only optical characteristics of electrodes and antireflection (AR) coatings deposited outside the active region of the device, thus reducing impacts on electrical characteristics and device complexity. The device used Mo having a high work function and moderate reflectivity as the bottom anode, and used the ultrathin LiF/Al bilayer and a thin $\mathrm{Ag}(\sim 20 \mathrm{~nm})$ as the electron-injection layer and the top semitransparent cathode, respectively. The device was further capped with one high-index dielectric layer $\left(\mathrm{TeO}_{2}\right.$, $n \sim 2.3-2.5$ ) and one low-index layer (LiF, $n \sim 1.3-1.4$ ) as the anti-reflection coatings. In such a device structure, the most pronounced reflections are from the bottom Mo mirror, the thin Ag mirror, and the surface of the high-index $\mathrm{TeO}_{2}$ [Fig. 29(a)]. By carefully adjusting phases of these three reflections with organic and dielectric thicknesses, one in principle can place two reflection minima at desired wavelengths in the reflection 


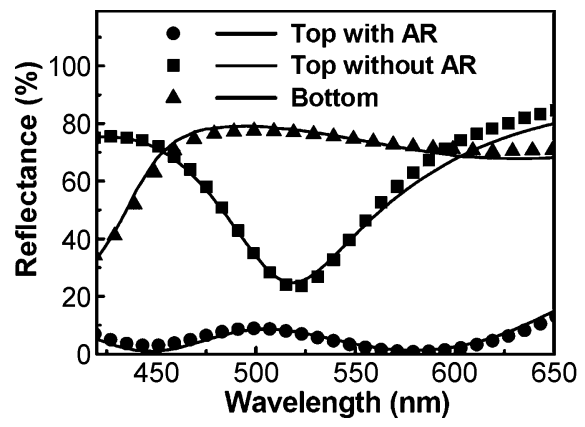

Fig. 30. Calculated (lines) and measured (symbols) reflectance spectra of the top-emitting OLEDs with and without AR coatings, and the conventional bottom-emitting OLEDs.

(a) Top

(b) Bottom

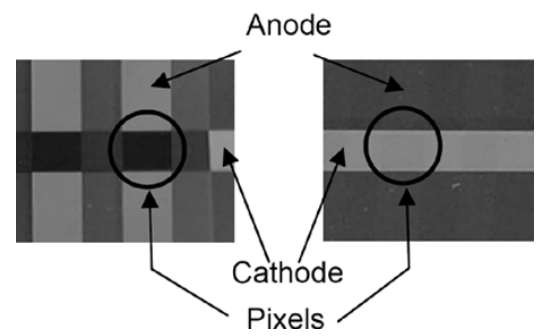

Fig. 31. Photos of: (a) the low-reflection top-emitting and (b) the conventional bottom-emitting OLEDs.

spectrum and produce suppressed reflectance over a wide band as one does with conventional arts of multilayer AR coatings [71], [72]. Fig. 30 shows the reflection spectrum of the optimized device, in comparison with those of a similar top-emitting device without the AR coatings [Fig. 29(b)] and a conventional bottom-emitting device. In the top-emitting devices with AR coatings, the organic/dielectric thicknesses were set to give two minima around 450 and $580 \mathrm{~nm}$ for obtaining a wide low-reflection band. The top-emitting device without AR coatings also exhibits reduced reflection in comparison with conventional devices, yet it is only over a limited spectral range, characteristic of a metal-dielectric-metal Fabry-Perot interferometer [71]. Overall, the high-contrast top-emitting device gives a luminous reflectance of $\sim 3.9 \%$, which is over one order lower than that of a conventional bottom-emitting device $(\sim 80 \%)$. Photos in Fig. 31 show the appearance of a low-reflection top-emitting OLED and a highly reflective bottom-emitting OLED under strong illumination, clearly indicating the dark background of the high-contrast top-emitting OLED.

Yang's low-reflection top-emitting device gave $I-V$ characteristics almost identical to those of a conventional bottom-emitting device [Fig. 32(a)], and achieved an cd/A efficiency over $60 \%$ of that of the bottom-emitting device [Fig. 32(b)]. Such efficiency performance represents a $\sim 1.5$ times enhancement compared to that obtained with the bottom-emitting OLED/polarizer combination, since the circular polarizer typically has a transmittance of only $\sim 40 \%$. The efficiency achieved is higher than those previous high-contrast devices and the enhancement may be attributed to the microcavity effect inherent in the device structure. Yang's high-contrast top-emitting OLEDs are readily compatible with the processing of active-matrix backplanes and had been implemented into a 3.8-in QVGA (i.e. $240 \times 320$ ) (a)

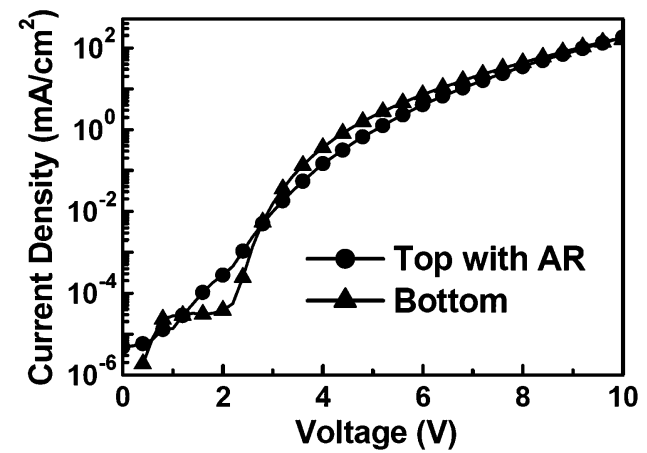

(b)

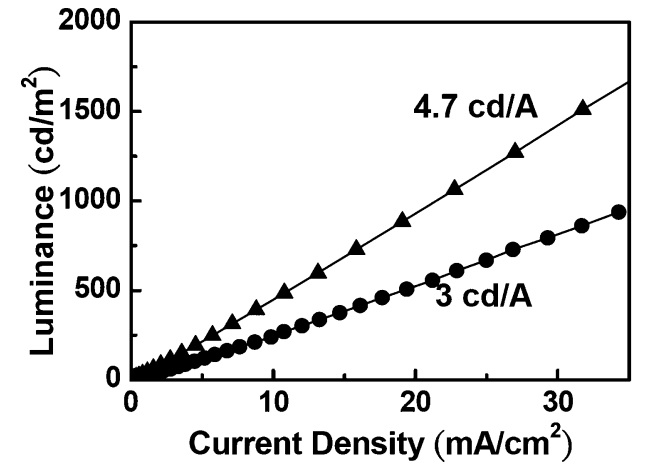

Fig. 32. (a) $I-V$ characteristics and (b) brightness-current characteristics of the low-reflection top-emitting OLED and the conventional bottom-emitting OLED.

(a)

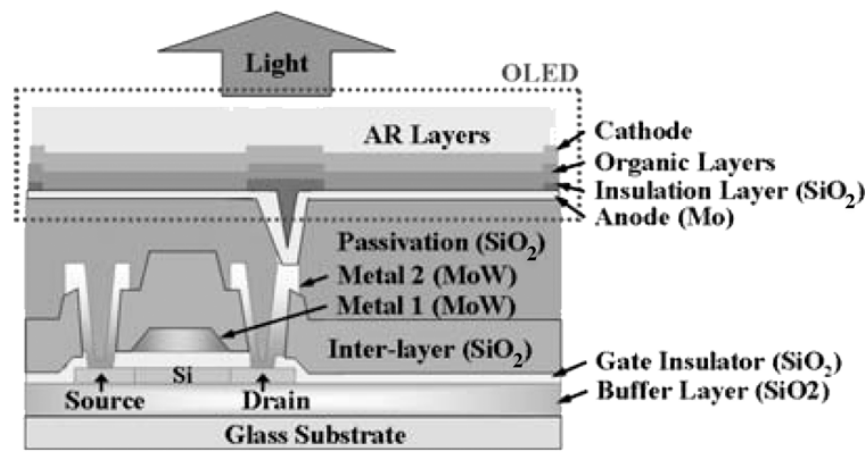

(b)

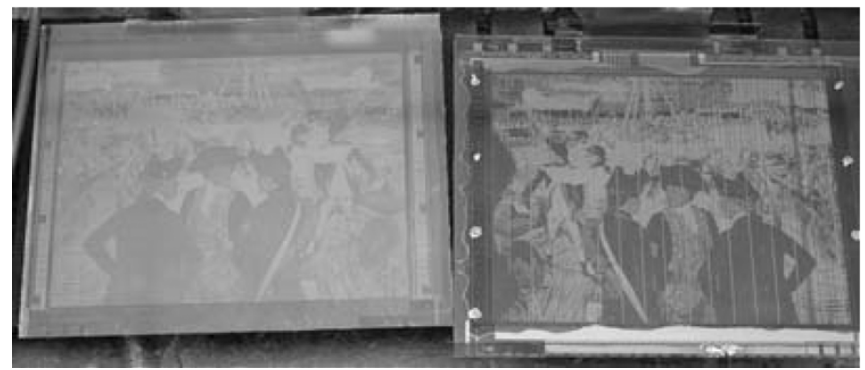

Fig. 33. (a) Cross section of the low-reflection top-emitting AMOLED and (b) photos of the low-reflection top-emitting (right) and the conventional bottom-emitting (left) AMOLEDs.

AMOLED with top-gate low-temperature polycrystalline silicon (LTPS) TFTs [Fig. 33(a)]. Fig. 33(b) shows the side-byside comparison of displayed images from the high-contrast topemitting AMOLED (right) and a conventional bottom-emitting 
(a)

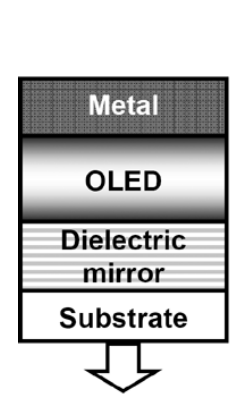

(d)

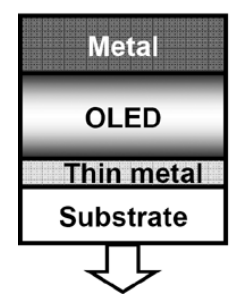

(b)

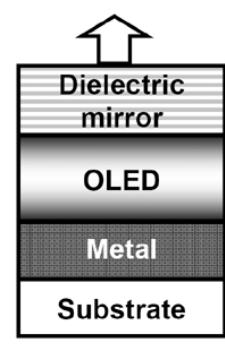

(e)

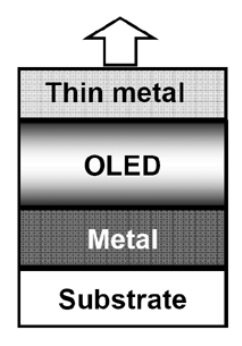

(c)

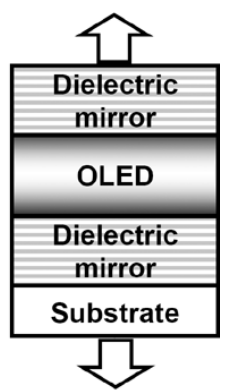

Fig. 34. Various structures of microcavity OLEDs employing different combinations of mirrors: (a), (b) using one metal mirror and one dielectric mirror; (c) using two dielectric mirrors; and (d), (e) using two metal mirrors.

AMOLED (left) under a strong lighting environment $(\sim 1000$ lux). While the high-contrast top-emitting AMOLED still exhibits an image of clear contrast, the conventional AMOLED shows strong reflection and image washout.

\section{MiCROCAVITY OLEDS}

As discussed in Section II, microcavity effects are inherent in any OLED structures. Microcavity OLEDs to be discussed in this section, however, are strong microcavity devices having electrode mirrors of strong reflection. As discussed in Section II, incorporation of strong microcavity structures in OLEDs can narrow emission spectra of devices and thus improve color purity for display applications. With appropriate microcavity designs, microcavity OLEDs may also give enhanced luminance and (cd/A) efficiency at least along the forward direction. Thus, microcavity OLEDs are becoming more attractive for high-performance AMOLEDs.

The mirrors for microcavity devices may be implemented with dielectric mirrors (distributed Bragg reflectors (DBRs) composed of alternating high/low-index layers) or reflective metals. Various possible structures of microcavity OLEDs employing different combinations of mirrors are shown in Fig. 34 [23], [24], [27], [41]-[44], [59], [73]-[77]. In the early development of microcavity OLEDs, Nakayama et al. and Dodabalapur et al. demonstrated microcavity bottom-emitting OLEDs with the reflective top metal electrode and the dielectric mirrors of $\mathrm{TiO}_{2} / \mathrm{SiO}_{2}$ or $\mathrm{Si}_{x} \mathrm{~N}_{y} / \mathrm{SiO}_{2}$ below the bottom ITO anode [i.e., the structure of Fig. 34(a)] [23], [24], [74], [75]. Narrowed EL emission spectra in comparison with intrinsic PL spectra of emitting materials were observed. Tsutsui et al. observed directed emission in microcavity bottom-emitting OLEDs with the structure of Fig. 34(a) [76]. In 1996, Jordan et al. were the first to show that by using one metal mirror and

one lossless dielectric mirror [i.e., the structure of Fig. 34(a)], microcavity OLEDs could give substantially enhanced luminance in comparison with noncavity (indeed weak-microcavity) OLEDs [24].

General complexity in the fabrication of dielectric mirrors and their strong wavelength-dependent reflection properties render them very difficult to be implemented into real OLED displays. In view of these, microcavity OLEDs using metal mirrors [one reflective and one semitransparent/reflective, Fig. 34(d) and (e)] are more practical for display applications [27], [41]-[44], [59], [77]. Indeed as briefly mentioned in previous sections, some top-emitting OLEDs having one reflective bottom metal electrode and one semitransparent top metal electrode usually exhibit rather strong microcavity effects, such as narrowed emission and directed emission, etc. However, due to absorption (loss) in metals, it is not clear whether one still can obtain luminance enhancement as in microcavity OLEDs using lossless dielectric mirrors, and also not clear to what degree and under what conditions one obtains most luminance enhancement.

Recently, Lin et al. performed detailed theoretical and experimental studies on the efficiencies of microcavity OLEDs using the semitransparent metal as the exit mirror [27]. As discussed in Section II of this article, to obtain maximal luminance enhancement (in the forward direction) in any microcavity OLEDs relative to conventional noncavity bottom-emitting devices, a back mirror with the highest possible reflectance and an exit mirror with a high reflectance matching that of the back electrode and a lowest possible loss are essential. Yet for an exit mirror using a semitransparent metal electrode, at the thickness of large enough reflectance, the absorptance, however, may become too large and degrade luminance enhancement. According to Lin's analysis, the exit mirror combining a thin metal and a transparent high-index dielectric layer may overcome such a dilemma. In their work, Lin et al. calculated the optical properties of a thin $\mathrm{Ag}$ film combined with the high-index $\mathrm{TeO}_{2}$ ( $n$ 2.3-2.5) as the composite mirror. Fig. 35(a) shows the calculated optical characteristics (reflectance $(\mathrm{R})$, transmittance (T), absorptance (A) at $520 \mathrm{~nm}$ ) viewed from a typical organic material for a thin $\mathrm{Ag}$ electrode $(24 \mathrm{~nm})$ with $\mathrm{TeO}_{2}$ of varied thickness. Ag is considered as it has relatively larger reflection, smaller absorption and higher conductivity among metals. Reflectance of $\mathrm{Ag} / \mathrm{TeO}_{2}$ is largely modulated with the $\mathrm{TeO}_{2}$ thickness, and for a certain range of thickness, reflectance is enhanced and absorptance is reduced down to $\sim 9 \%$ (versus $15 \%$ with no $\mathrm{TeO}_{2}$ ). In this case, to search for combinations of $\mathrm{Ag} / \mathrm{TeO}_{2}$ giving optimal luminance enhancement from a microcavity device, it is useful to construct a contour plot of $G_{\text {int }}$ versus $\mathrm{Ag}$ and $\mathrm{TeO}_{2}$ thicknesses (Fig. 35(b)). For a certain range of $\mathrm{Ag} / \mathrm{TeO}_{2}$ combinations, one obtains maximal $G_{\text {int }}$ of 2.4-2.6. Detailed analyses (comparing Fig. 3(b), Fig. 35(c), and Fig. 35(d)) reveal that these conditions are roughly coincident with those giving high reflection and low absorption.

Lin et al. also conducted experiments on microcavity top-emitting OLED with a nearly optimized structure of: glass/Ag (80 nm)/m-MTDATA:F4-TCNQ (2 wt.\%, 20 $\mathrm{nm}) / \alpha$-NPD $(25 \mathrm{~nm}) / \mathrm{Alq}_{3}:$ C545T (1 wt.\%, $\left.20 \mathrm{~nm}\right) / \mathrm{Alq}_{3}$ $(35 \mathrm{~nm}) / \mathrm{LiF}(0.5 \mathrm{~nm}) / \mathrm{Al}(1 \mathrm{~nm}) / \mathrm{Ag}(24 \mathrm{~nm}) / \mathrm{TeO}_{2}(55 \mathrm{~nm})$, 
(a)

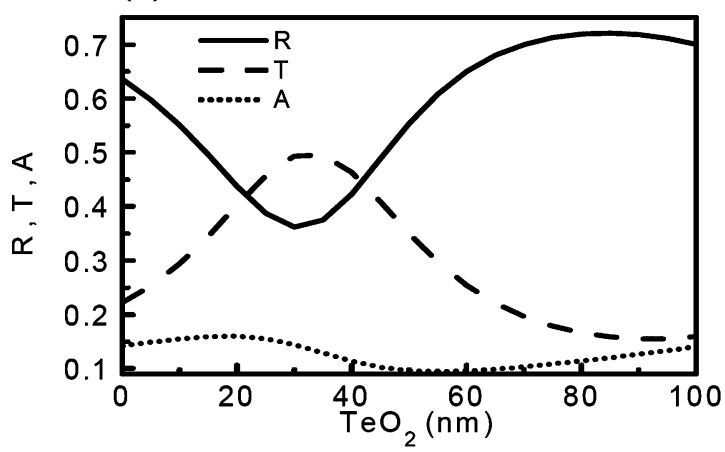

(b) $\mathrm{G}_{\text {int }}$

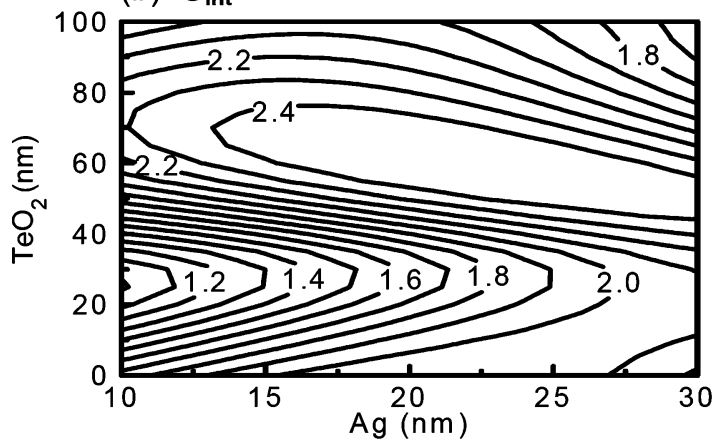

(c) $\mathrm{R}$

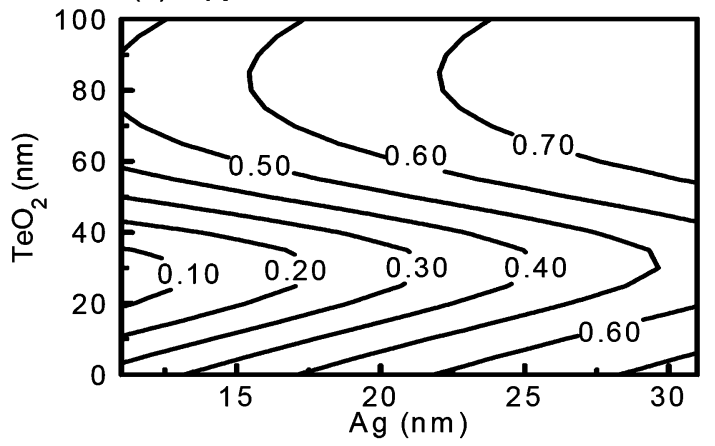

(d) $\quad A$

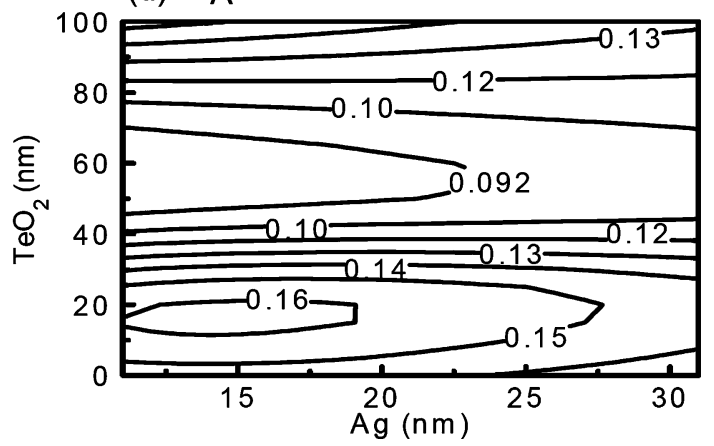

Fig. 35. (a) Calculated $R, T, A$ (at $520 \mathrm{~nm}$ ) of the composite mirror of thin $\mathrm{Ag}(24 \mathrm{~nm})$ and $\mathrm{TeO}_{2}$. (b) Contour plot of calculated $G_{\text {int }}$ versus $\mathrm{Ag}$ and $\mathrm{TeO}_{2}$ thicknesses for the optimized microcavity OLED having a back mirror with a reflectance of 0.9 , and having an emitter with $\lambda_{\text {em }}=520 \mathrm{~nm}, \Delta \lambda_{\text {em }}=60 \mathrm{~nm}$, $\tau_{\text {cav }} / \tau_{\text {con }}=1$. (c) Contour plot of $R$ (at $520 \mathrm{~nm}$ ) of the composite mirror of thin $\mathrm{Ag}(24 \mathrm{~nm})$ and $\mathrm{TeO}_{2}$. (d) Contour plot of $A$ (at $520 \mathrm{~nm}$ ) of the composite mirror of thin $\mathrm{Ag}(24 \mathrm{~nm})$ and $\mathrm{TeO}_{2}$.

in which $\mathrm{Ag}$ and $\mathrm{Ag} / \mathrm{TeO}_{2}$ serve as the bottom anode and the top cathode, respectively. Other layers in sequence consist of m-MTDATA doped with 2 wt.\% of F4-TCNQ as the hole-injection layer, $\alpha$-naphthylphenylbiphenyl diamine ( $\alpha$-NPD) as (a)

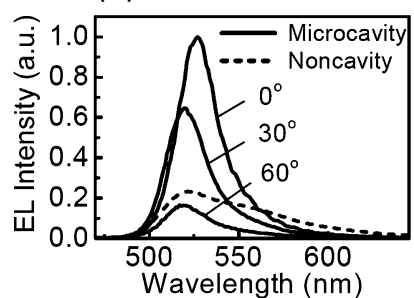

(c)

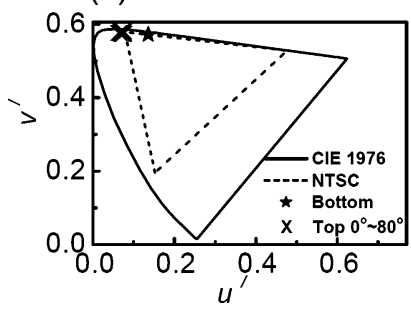

(b)

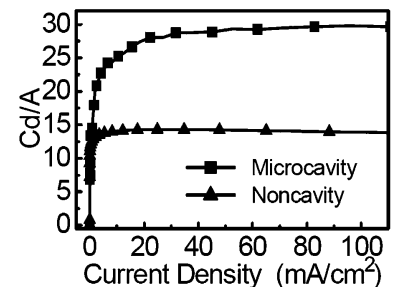

(d)

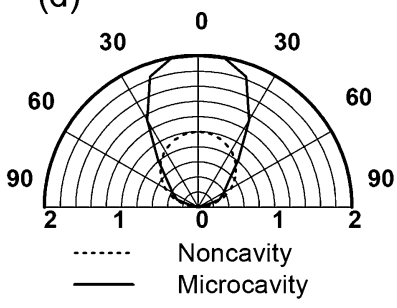

Fig. 36. Comparison of optical characteristics of a microcavity device and a noncavity device. (a) Measured EL spectra with relative intensities at $0^{\circ}$, $30^{\circ}$, and $60^{\circ}$ off the surface normal of the microcavity device, and at $0^{\circ}$ of the noncavity device. (b) cd/A efficiencies for both devices. (c) 1976 CIE coordinates of $0^{\circ}-80^{\circ}$ EL of the microcavity device and $0^{\circ}$ EL of the noncavity device. (d) Polar plots of measured EL intensities (normalized to the $0^{\circ}$ intensity of the noncavity device) for both devices.

the hole-transport layer, $\mathrm{Alq}_{3}$ doped with the fluorescent dye C545T $\left(\Delta \lambda_{\mathrm{em}} \approx 60 \mathrm{~nm}\right)$ as the emitting layer, undoped $\mathrm{Alq}_{3}$ as the electron-transport layer, and thin $\mathrm{LiF} / \mathrm{Al}$ as the electron injection layer. For comparison, an optimized noncavity bottom-emitting device was also fabricated with the structure of: glass/ITO (120 nm)/m-MTDATA:F4-TCNQ (2 wt.\%, 20 $\mathrm{nm}) / \alpha$-NPD $(20 \mathrm{~nm}) / \mathrm{Alq}_{3}:$ C545T (1 wt.\%, $\left.20 \mathrm{~nm}\right) / \mathrm{Alq}_{3}(40$ $\mathrm{nm}) / \mathrm{LiF}(0.5 \mathrm{~nm}) / \mathrm{Al}(1 \mathrm{~nm}) / \mathrm{Ag}(150 \mathrm{~nm})$. In both devices, layer thicknesses had been determined based on the antinode and resonance conditions near $\lambda_{\mathrm{em}}$ of C545T (520 nm). Fig. 36(a) shows the measured electroluminescence (EL) spectra with relative intensities at viewing angles of $0^{\circ}, 30^{\circ}$, and $60^{\circ}$ off the surface normal of the microcavity device, and at $0^{\circ}$ of the noncavity device. The microcavity device shows a forward $\mathrm{G}_{\text {int }}$ of $\sim 2.0$ (and an enhancement of $\sim 4.3$ at the $0^{\circ}$ resonance wavelength). Correspondingly, the microcavity device gives a 2.0 times larger cd/A efficiency than the noncavity device [30 cd/A versus $14.2 \mathrm{~cd} / \mathrm{A}$, Fig. 36(b)]. Fig. 36(c) shows the CIE coordinates (1976 uniform color space) of $0^{\circ}-80^{\circ} \mathrm{EL}$ of the microcavity device and that of the noncavity device. The microcavity device shows more saturated colors than the NTSC green standard and negligible color shift with viewing angles, both the desired characteristics for displays. Fig. 36(d) compares the angular distributions of EL intensity (normalized to the $0^{\circ}$ intensity of the noncavity device) for both devices. The microcavity device shows more directed emission and enhanced luminance for $0^{\circ}-45^{\circ}$. Such characteristics shall be an advantage for small- or medium-sized OLED displays, which are mainly viewed from the forward direction. The external EL quantum efficiency for the cavity device is slightly higher than that of the noncavity device $(4.1 \%$ versus $3.8 \%)$, indicating the forward-direction enhancement results mainly from redistribution of emission directions. 
(a)

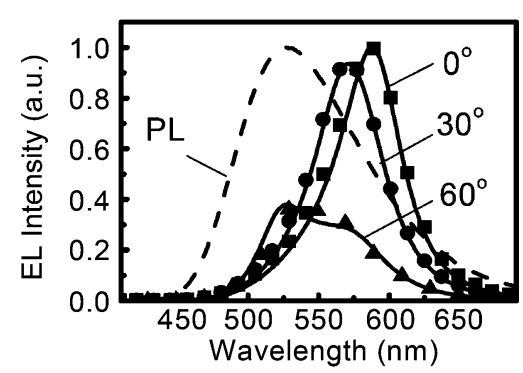

(b)

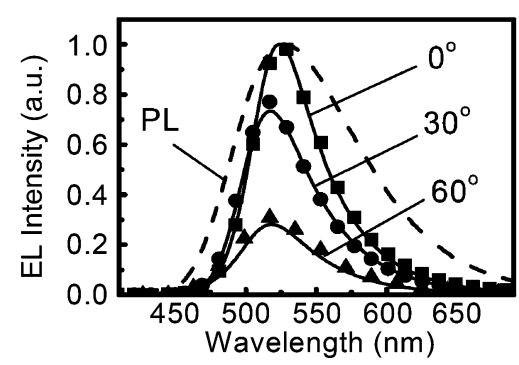

Fig. 37. Measured (solid symbols) and calculated (solid lines) EL spectra with relative intensities at viewing angles of $0^{\circ}, 30^{\circ}$, and $60^{\circ}$ for: (a) the microcavity device with the forward resonant wavelength set at $\sim 585 \mathrm{~nm}$ and (b) the microcavity device with the forward resonant wavelength set at $\sim 520$ $\mathrm{nm}$, in comparison with the PL spectrum of the emitter $\mathrm{Alq}_{3}$.

Another important issue associated with microcavity OLEDs is the dependence of emission spectra and colors on viewing angles. Similar to (1) in Section II-A, one can also derive the enhancement factor for waves propagate along some internal angle $\theta$ off the surface normal of the microcavity for each polarization [78]. At a certain oblique direction $\theta$, the resonant wavelength $\lambda_{m}$ of the Fabry-Perot cavity is

$$
\lambda m=\frac{4 \pi n L \cos \theta}{\phi_{1}+\phi_{2}-2 \pi m} .
$$

Definitions of $\phi_{1}$ and $\phi_{2}$ are similar to those in (1), except that they now may have dependence on $\theta$ and polarization. Yet, in general, the resonant wavelength and the peak position of EL spectra of microcavity OLEDs shift to shorter wavelengths with viewing angles. Such a color shift with viewing angles would degrade the viewing characteristics in display applications. Recently, Lin et al. performed systematic studies on such effects and suggested appropriate microcavity designs for remedy [31]. Using the strong microcavity OLED structure consisting of two metal mirrors and the $\mathrm{Alq}_{3}$ emitter, Lin et al. performed analysis using the rigorous electromagnetic modeling as described in Section II-B and verified analysis with experiments. It was found that, in designing the microcavity structure, if the resonant wavelength is set larger than the peak wavelength of the intrinsic emission (PL) spectrum of the emitter, EL spectra from the microcavity OLED will exhibit a large blue shift with viewing angles [Fig. 37(a)]. Yet, if the resonant wavelength is set equal to or smaller than the peak wavelength of the intrinsic spectrum of the emitter, EL spectra from the microcavity OLED will exhibit hardly detectable color shift with viewing angles [Fig. 37(b)]. In the latter case [Fig. 37(b)], although the EL spectra tend to blue shift according to (3), yet the falloff at the shorter-wavelength

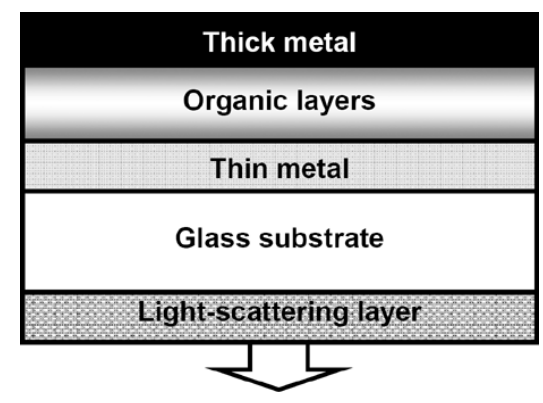

Fig. 38. Schematic diagram showing the structure of a broadband microcavity OLED.

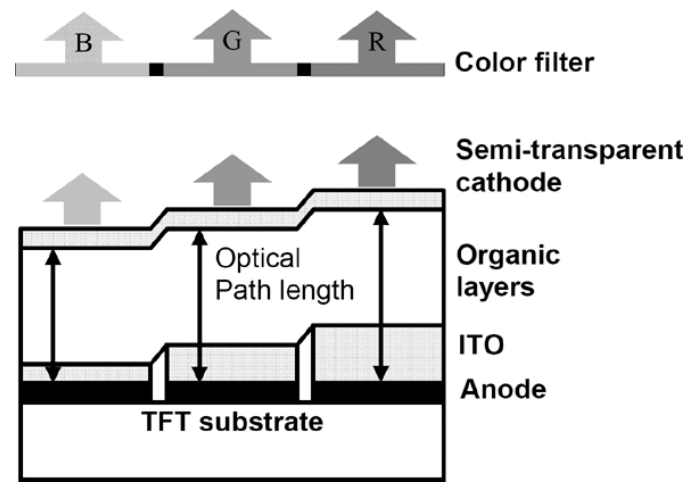

Fig. 39. The structure of the AMOLED demonstrated by Sony Corporation in SID 2004 incorporating top-emitting microcavity white OLEDs and color filters. The different microcavity length required each color sub-pixel is controlled by the thickness of ITO overcoating on the reflective bottom anode.

half of the intrinsic emission spectrum constraints such a shift, and thus gives hardly detectable color shifts.

The spectral shift with viewing angles due to the miccrocavity indeed may have some nice applications. In SID 2005 [79], Tyan et al. demonstrated a broadband bottom-emitting microcavity OLED by laminating a light-scattering (diffuser) film onto the surface of the microcavity device having two metal mirrors (Fig. 38). The diffuser film remixes emission (of different colors) from different angles of the microcavity OLED and redistributes emission into different angles, giving broadband emission over all angles. In addition, Tyan et al. found that the addition of the diffuser film also helps to extract more short-wavelength light from the substrate, that otherwise would be seriously trapped due to total internal reflection. Although such an approach may not be suitable for display applications which prefers narrow emitters, Tyan et al. suggested it may find nice applications in lighting devices, where broadband emitters with high efficiency are welcome.

In addition to using microcavity structures for enhancing color purity and luminance of displays, in SID 2004 [80], Sony Corporation demonstrated the use of microcaity OLEDs in enhancing display contrast. Fig. 39 shows the implementation of the display structure, which combines top-emitting miccrocavity white OLEDs and color filters. Since the stack structures of organic layers are the same for all three sub-pixels, the different microcavity length required for each color sub-pixel is controlled by the thickness of ITO overcoating the reflective bottom electrode. The combination of microcavity structures and color filters results in significant enhancement of the 


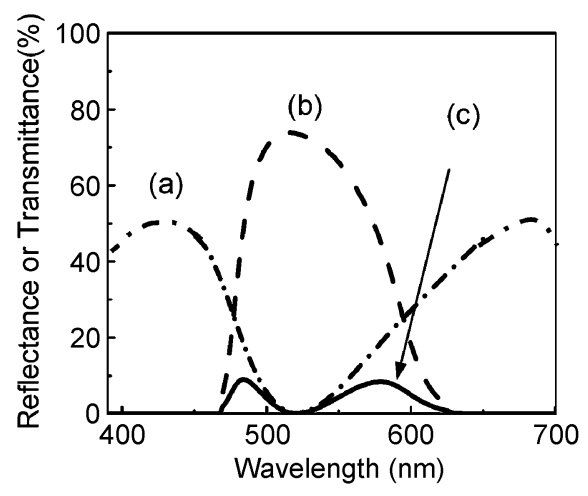

Fig. 40. Illustration of the combination of the microcavity structure and the color filter for enhancing display contrast (reducing reflection): (a) reflection spectrum of the microcavity; (b) transmission spectrum of the color filter; and (c) reflection spectrum of the microcavity combined with the color filter.

contrast due to the matching of the low-reflection band of the microcavity and the high-transmission band of the color filter, as shown in Fig. 40 for the (green) sub-pixel. The microcavity OLED for the (green) sub-pixel is tuned to have resonance for green emission, and correspondingly gives low reflection in the similar band. Such a characteristic in combination with strong absorption outside the transmission band (also green) of color filters naturally gives the complete pixel low reflection and high contrast over the whole visible range. The very advantage of such a contrast-enhancement technique is that, unlike conventional techniques, the contrast is largely enhanced without significantly sacrificing emission efficiency.

\section{SUMMARY}

In summary, several advanced OLED structures that are useful for enhancing performances of AMOLED displays, such as top-emitting OLEDs, inverted OLEDs, high-contrast OLEDs, and microcavity OLEDs, have been reviewed. General discussions of the optical structures and optical characteristics of OLEDs based on analytical Fabry-Perot formulations and rigorous electromagnetic modeling are first given and then used as the basis in discussions of optical characteristics of each advanced OLED structure. In general, optically an OLED can be considered as a microcavity, either weak or strong. To optimize optical performances of devices such as efficiencies and colors etc., the resonance conditions must be appropriately set. The article then reviews the development of these advanced OLED structures, starting with their technical purposes and then concentrating on major technical issues associated with each OLED technology, such as how to prepare electrodes with desired electrical and optical properties and how to design their optical structures. The discussions of each OLED technology then conclude with its applications in AMOLEDs. Top-emitting OLEDs have been used in AMOLEDs for increasing aperture ratios of pixels. Inverted OLEDs render feasible implementation of AMOLEDs using higher-performance n-type transistors in pixel circuits. High-contrast OLED displays may be realized using high-contrast OLEDs to reduce impact on cost and fabrication. Finally, incorporation of microcavity structures in any type of OLEDs could be used to enhance color purity, brightness, and efficiency of OLED displays.

\section{REFERENCES}

[1] W. Helfrich and W. G. Schneider, "Recombination radiation in anthracene crystals," Phys. Rev. Lett., vol. 14, no. 7, pp. 229-231, Feb. 1965.

[2] M. Pope, H. Kallman, and P. Magnante, "Electroluminescence in organic crystals," J. Chem. Phys., vol. 38, no. 8, p. 2042, Apr. 1963.

[3] C. W. Tang and S. A. Vanslyke, "Organic electroluminescent diodes," Appl. Phys. Lett., vol. 51, no. 12, pp. 913-915, Sep. 1987.

[4] C. W. Tang, S. A. Vanslyke, and C. H. Chen, "Electroluminescence of doped organic thin films," J. Appl. Phys., vol. 65, no. 9, pp. 3610-3616, May 1989.

[5] J. H. Burreughes, D. D. C. Bredly, A. R. Brown, R. N. Marks, K. Mackay, and R. H. Friend, Nature (London), vol. 347, no. 6293, p. 539, Oct. 1990.

[6] T. Wakimoto, R. Murayama, K. Nagayama, Y. Okuda, H. Nakada, and T. Tohma, "Organic LED dot-matrix display," in Dig. Tech. Papers of 1996 Int. Symp. Soc. of Inf. Display, 1996, pp. 849-852.

[7] R. M. A. Dawson, Z. Shen, D. A. Furst, S. Connor, J. Hsu, M. G. Kane, R. G. Stewart, A. Ipri, C. N. King, P. J. Green, R. T. Flegal, S. Pearson, W. A. Barrow, E. Dickey, K. Ping, C. W. Tang, S. Van Slyke, F. Chen, J. Shi, J. C. Sturm, and M. H. Lu, "Design of an improved pixel for a polysilicon active-matrix organic LED display," in Dig. Tech. Papers of 1998 Int. Symp. Soc. of Inf. Display, 1996, pp. 11-14.

[8] R. M. A. Dawson, Z. Shen, D. A. Furst, S. Connor, J. Hsu, M. G. Kane, R. G. Stewart, A. Ipri, C. N. King, P. J. Green, R. T. Flegal, S. Pearson, W. A. Barrow, E. Dickey, K. Ping, S. Robinson, C. W. Tang, S. Van Slyke, F. Chen, J. Shi, M. H. Lu, M. Moskewicz, and J. C. Sturm, "A poly-Si active-matrix OLED display with integrated drivers," in Dig. Tech. Papers of 1999 Int. Symp. Soc. of Inf. Display, 1999, pp. 11-14.

[9] R. M. A. Dawson and M. G. Kane, "Pursuit of active matrix organic light-emitting diode displays," in Dig. Tech. Papers of 2001 Int. Symp. Soc. of Inf. Display, 2001, pp. 372-375.

[10] T. Shimoda, M. Kimura, S. Seki, H. Kobayashi, S. Kanbe, S. Miyashita, R. H. Friend, J. H. Burroughes, C. R. Towns, and I. S. Millard, "Technology for active matrix light emitting polymer displays," in Tech. Dig. 1999 IEEE Int. Electron Devices Meeting, 1999, pp. 573-576.

[11] K. Inuki, H. Kimura, M. Mizukami, J. Maruyama, S. Murakami, J. Koyama, T. Konuma, and S. Yamazaki, "4.0-in TFT-OLED displays and a novel digital driving methode," in Dig. Tech. Papers of 2000 Int. Symp. Soc. of Inf. Display, 2000, pp. 924-927.

[12] Y. He, R. Hattori, and J. Kanicki, "Current-source a-Si:H thin-film transistor circuit for active-matrix organic light-emitting displays," IEEE Electron. Dev. Lett., vol. 21, no. 12, pp. 590-592, Dec. 2000.

[13] G. W. Jones, "Active matrix OLED microdisplays," in Dig. Tech. Papers of 2001 Int. Symp. Soc. of Inf. Display, 2001, pp. 134-137.

[14] T. Sasaoka, M. Sekiya, A. Yumoto, J. Yamada, T. Hirano, Y. Iwase, T. Yamada, T. Ishibashi, T. Mori, M. Asano, S. Tamura, and T. Urabe, "A 13.0-inch AM-OLED display with top emitting structure and adaptive current mode programmed pixel circuit (TAC)," in Dig. Tech. Papers of 2001 Int. Symp. Soc. of Inf. Display, 2001, pp. 384-387.

[15] M. A. Baldo, D. F. O’Brien, Y. You, A. Shoustikov, S. Sibley, M. E. Thompson, and S. R. Forrest, "Highly efficient phosphorescent emission from organic electroluminescent devices," Nature (London), vol. 395, no. 6698, pp. 151-154, Sep. 1998.

[16] L. S. Hung and C. H. Chen, "Recent progress of molecular organic electroluminescent materials and devices," Mater. Sci. Eng. R, vol. R39, no. 5-6, pp. 143-222, Dec. 2002.

[17] J.-J. Lih, C.-F. Sung, M. S. Weaver, M. Hack, and J. J. Brown, “A phosphorescent active-matrix OLED display driven by amorphous silicon backplane," in Dig. Tech. Papers of 2003 Int. Symp. Soc. of Inf. Display, 2003, pp. 14-17.

[18] J. Y. Lee, J. H. Kwon, and H. K. Chung, "High efficiency and low power consumption in active matrix organic light emitting diodes," Org. Electron., vol. 4, no. 2-3, pp. 143-148, Sep. 2003.

[19] K. A. Neyts, "Simulation of light emission from thin-film microcavities," J. Opt. Soc. Amer. A, vol. 15, no. 4, pp. 962-971, Apr. 1998.

[20] H. Riel, S. Karg, T. Beierlein, W. Ries, and K. Neyts, "Tuning the emission characteristics of top-emitting organic light-emitting devices by means of a dielectric capping layer: an experimental and theoretical study," J. Appl. Phys., vol. 94, no. 8, pp. 5290-5296, Oct. 2003. 
[21] Z. Huang, C. Lei, D. G. Deppe, C. C. Lin, C. J. Pinzone, and R. D. Dupuis, "Spectral and intensity dependence on dipole localization in Fabry-Perot cavities," Appl. Phys. Lett., vol. 61, no. 25, pp. 2961-2963, Dec. 1992.

[22] E. F. Schubert, N. E. J. Hunt, M. Micovic, R. J. Malik, D. L. Sivco, A. Y. Cho, and G. J. Zydzik, "Highly efficient light-emitting diodes with microcavities," Science, vol. 265, no. 5174, pp. 943-945, Aug. 1994.

[23] A. Dodabalapur, L. J. Rothberg, R. H. Jordan, T. M. Miller, R. E. Slusher, and J. M. Phillips, "Physics and applications of organic microcavity light-emitting diodes," J. Appl. Phys., vol. 80, no. 12, pp. 6954-6964, Dec. 1996.

[24] R. H. Jordan, L. J. Rothberg, A. Dodabalapur, and R. E. Slusher, "Efficiency enhancement of microcavity organic light-emitting diodes," Appl. Phys. Lett., vol. 69, no. 14, pp. 1997-1999, Sep. 1996.

[25] G. R. Hayes, F. Cacialli, and T. R. Phillips, "Ultrafast study of spontaneous emission from conjugated polymer microcavities," Phys. Rev. B, vol. 56, no. 8, p. 4798, Aug. 1997.

[26] U. Lemmer, R. Hennig, W. Guss, A. Ochse, J. Pommerehne, R. Sander, A. Greiner, R. F. Mahrt, H. Bässler, J. Feldmann, and E. O. Göbel, "Microcavity effects in a spin-coated polymer two-layer system," Appl. Phys. Lett., vol. 66, no. 11, p. 1301, Mar. 1995.

[27] C.-L. Lin, H.-W. Lin, and C.-C. Wu, "Examining microcavity organic light-emitting devices having two metal mirrors," Appl. Phys. Lett., vol. 87, no. 2, p. 021101, Jul. 2005.

[28] W. Lukosz and R. E. Kunz, "Light-emission by magnetic and electric dipoles close to a plane interface I: total radiation power," J. Opt. Soc. Am., vol. 67, no. 12, pp. 1607-1615, Dec. 1977.

[29] J. E. Sipe, "The dipole antenna problem in surface physics-a new approach," Surf. Sci., vol. 105, no. 2-3, pp. 489-504, Nov. 1981.

[30] J. A. E. Wasey and W. L. Barnes, "Efficiency of spontaneous emission from planar microcavities," J. Mod. Opt., vol. 47, no. 4, pp. 725-741, Mar. 2000.

[31] C.-C. Wu, C.-L. Lin, P.-Y. Hsieh, and H.-H. Chiang, "Methodology for optimizing viewing characteristics of top-emitting organic light-emitting devices," Appl. Phys. Lett., vol. 84, no. 20, pp. 3966-3968, May 2004.

[32] G. Gu, V. Bulovic, P. E. Burrows, S. R. Forrest, and M. E. Thompson, "Transparent organic light-emitting devices," Appl. Phys. Lett., vol. 68, no. 19, pp. 2606-2608, May 1996.

[33] P. E. Burrows, G. Gu, S. R. Forrest, E. P. Vincezi, and T. X. Zhou, "Semitransparent cathodes for organic light-emitting devices," J. Appl. Phys., vol. 87, no. 6, pp. 3080-3085, Mar. 2000.

[34] T. X. Zhou, J. J. Brown, J. K. Mahon, P. E. Burrows, and S. R. Forrest, "Optimization of reliable transparent organic LEDs," in Dig. Tech. Papers of 1999 Int. Symp. Soc. of Inf. Display, 1999, pp. 434-437.

[35] G. Parthasarathy, P. E. Burrows, V. Khalfin, V. G. Kozlov, and S. R. Forrest, "A metal-free cathode for organic semiconductor devices," Appl. Phys. Lett., vol. 72, no. 17, pp. 2138-2140, Apr. 1998.

[36] L. S. Hung and C. W. Tang, "Interface engineering in preparation of organic surface-emitting diodes," Appl. Phys. Lett., vol. 74, no. 21, pp. 3209-3211, May 1999.

[37] G. Parthasarathy, C. Adachi, P. E. Burrows, and S. R. Forrest, "Highefficiency transparent organic light-emitting devices," Appl. Phys. Lett., vol. 76, no. 25, pp. 2128-2130, Apr. 2000.

[38] H. Kanno, Y. Sun, and S. R. Forrest, "High-efficiency top-emissive white-light-emitting organic electrophosphorescent devices," Appl. Phys. Lett., vol. 86, no. 26, p. 263502, Jun. 2005.

[39] A. Yamamori, S. Hayashi, T. Koyama, and Y. Taniguchi, "Transparent organic light-emitting diodes using metal acethylacetonate complexes as an electron injective buffer layer," Appl. Phys. Lett., vol. 78, no. 21, pp. 3343-3345, May 2001.

[40] L. S. Hung, C. W. Tang, M. G. Mason, P. Raychaudhuri, and J. Madathil, "Application of an ultrathin $\mathrm{LiF} / \mathrm{Al}$ bilayer in organic surface-emitting diodes," Appl. Phys. Lett., vol. 78, no. 4, pp. 544-546, Jan. 2001.

[41] M.-H. Lu, M. S. Weaver, T. X. Zhou, M. Rothman, R. C. Kwong, M. Hack, and J. J. Brown, "High-efficiency top-emitting organic light-emitting devices," Appl. Phys. Lett., vol. 81, no. 21, pp. 3921-3923, Nov. 2002.

[42] H. Riel, S. Karg, T. Beierlein, B. Ruhstaller, and W. Rieß, "Phosphorescent top-emitting organic light-emitting devices with improved light outcoupling," Appl. Phys. Lett., vol. 82, no. 3, pp. 466-468, Jan. 2003.

[43] C.-W. Chen, P.-Y. Hsieh, H.-H. Chiang, C.-L. Lin, H.-M. Wu, and C.-C. $\mathrm{Wu}$, "Top-emitting organic light-emitting devices using surface-modified Ag anode," Appl. Phys. Lett., vol. 83, no. 25, pp. 5127-5129, Dec. 2003.
[44] S.-F. Hsu, C.-C. Lee, S.-W. Haung, and C. H. Chen, "Highly efficient top-emitting white organic electroluminescent devices," Appl. Phys. Lett., vol. 86, no. 25, p. 253508, Jun. 2005.

[45] S. M. Sze, Physics of Semiconductor Devices. New York: Wiley, 1981, ch. 5 .

[46] T. A. Beierlein, E. Haskal, H. Riel, W. Riess, P. Seidler, S. C. Strite, and H. Vestweber, "Anode modification for organic light-emitting diodes," U.S. Patent 6501217 B2, Dec. 31, 2002.

[47] C. Qiu, H. Peng, H. Chen, Z. Xie, M. Wong, and H.-S. Kwok, "Topemitting organic light-emitting diode using nanometer platinum layers as hole injector," in Dig. Tech. Papers of 2003 Int. Symp. Soc. of Inf. Display, 2003, pp. 974-977.

[48] Z. Xie, L.-S. Hung, and F. Zhu, "A flexible top-emitting organic lightemitting diode on steel foil," Chem. Phys. Lett., vol. 381, no. 5-6, pp. 691-696, Nov. 2003.

[49] K. H. Lee, S.-Y. Ryu, J. H. Kwon, S. W. Kim, and H. K. Chung, "2.2" QCIF full color transparent AMOLED display," in Dig. Tech. Papers of 2003 Int. Symp. Soc. of Inf. Display, 2003, pp. 104-107.

[50] Dig. Tech. Papers of 2004 Int. Symp. Soc. of Inf. Display, 2004, pp. 1403-1405.

[51] C.-W. Ko, S.-H. Hu, S.-H. Li, T.-H. Hsiao, K.-S. Lee, C.-J. Chen, and J.-J. Lih, "Development of 1.5-inch full-color double-sided AMOLED with novel array design," in Dig. Tech. Papers of 2005 Int. Symp. Soc. of Inf. Display, 2005, pp. 961-964.

[52] Dig. Tech. Papers of 2005 Int. Symp. Soc. of Inf. Display, 2005, pp. 1546-1549.

[53] D. R. Baigent, R. N. Marks, N. C. Greenham, R. H. Friend, S. C. Moratti, and A. B. Holmes, "Conjugated polymer light-emitting diodes on silicon substrates," Appl. Phys. Lett., vol. 65, no. 21, pp. 2636-2638, Nov. 1994

[54] V. Bulović, P. Tian, P. E. Burrows, M. R. Gokhale, and S. R. Forrest, "A surface-emitting vacuum-deposited organic light emitting devices," Appl. Phys. Lett., vol. 70, no. 22, pp. 2954-2956, Jun. 1997.

[55] T. Dobbertin, M. Kroeger, D. Heithecker, D. Schneider, D. Metzdorf, H. Neuner, E. Becker, H.-H. Johannes, and W. Kowalsky, "Inverted topemitting organic light-emitting diodes using sputter-deposited anodes," Appl. Phys. Lett., vol. 82, no. 2, pp. 284-286, Jan. 2003.

[56] T. Dobbertin, O. Werner, J. Meyer, A. Kammoun, D. Schneider, T. Riedl, E. Becker, H.-H. Johannes, and W. Kowalsky, "Inverted hybrid organic light-emitting device with polyethylene dioxythiophene-polystyrene sulfonate as an anode buffer layer," Appl. Phys. Lett., vol. 83, no. 24, pp. 5071-5703, Dec. 2003.

[57] X. Zhou, M. Pfeiffer, J. S. Huang, J. Blochwitz-Nimoth, D. S. Qin, A. Werner, J. Drechsel, B. Maennig, and K. Leo, "Low-voltage inverted transparent vacuum deposited organic light-emitting diodes using electrical doping," Appl. Phys. Lett., vol. 81, no. 5, pp. 922-924, Jul 2002.

[58] M. Pfeiffer, S. R. Forrest, X. Zhou, and K. Leo, "A low drive voltage, transparent, metal-free n-i-p electrophosphorescent light emitting diode," Org. Electron., vol. 4, no. 1, pp. 21-26, Jun. 2003.

[59] C.-W. Chen, C.-L. Lin, and C.-C. Wu, "An effective cathode structure for inverted top-emitting organic light-emitting devices," Appl. Phys. Lett., vol. 85, no. 5, pp. 2469-2471, Jul. 2004.

[60] H. Murakami, M. Shimizu, S. Aratani, and M. Tanaka, "High-efficiency and high-operational-stability top-emitting OLEDs," in Dig. Tech. Papers of 2005 Int. Symp. Soc. of Inf. Display, 2005, pp. 155-158.

[61] C. Prat, D. Vaufrey, C. Fery, J. Birnstock, M. Hofmann, P. Wellmann, Y.-H. Yeh, Y.-R. Liu, L.-C. Lin, and B.-C. Chang, "High-performance innovative OLED structure," in Dig. Tech. Papers of 2005 Int. Symp. Soc. of Inf. Display, 2005, pp. 151-154.

[62] L. S. Hung and J. Madathil, "Reduction of ambient light reflection in organic light-emitting diodes," Adv. Mater., vol. 13, no. 23, pp. 1787-1790, Dec. 2001.

[63] A. N. Krasnov, "High-contrast organic light-emitting diodes on flexible substrates," Appl. Phys. Lett., vol. 80, no. 20, pp. 3853-3855, May 2002.

[64] F. L. Wong, M. K. Fung, X. Jiang, C. S. Lee, and S. T. Lee, "Non-reflective black cathode in organic light-emitting diode," Thin Solid Films, vol. 446, no. 1, pp. 143-146, Jan. 2004.

[65] H. Aziz, Y.-F. Liew, H. M. Grandin, and Z. D. Popovic, "Reduced reflectance cathode for organic light-emitting devices using metalorganic mixtures," Appl. Phys. Lett., vol. 83, no. 1, pp. 186-188, Jul. 2003.

[66] Z. Y. Xie and L. S. Hung, "High-contrast organic light-emitting diodes," Appl. Phys. Lett., vol. 84, no. 7, pp. 1207-1209, Feb. 2004.

[67] X. D. Feng, R. Khangura, and Z. H. Lu, "Metal-organic-metal cathode structure for high-contrast organic light-emitting diodes," Appl. Phys. Lett., vol. 85, pp. 497-499, 2004.

[68] S. H. Li, H. Liem, C. W. Chen, E. H. Wu, Z. Xu, and Y. Yang, "Stacked metal cathode for high-contrast-ratio polymeric light-emitting devices," Appl. Phys. Lett., vol. 86, no. 14, p. 143514, Apr. 2005. 
[69] C.-J. Yang, C.-L. Lin, C.-C. Wu, Y.-H. Yeh, C.-C. Cheng, Y.-H. Kuo, and T.-H. Chen, "High-contrast top-emitting organic light-emitting devices for active-matrix displays," Appl. Phys. Lett., vol. 87, no. 14, p. 143507, Oct. 2005.

[70] Y.-H. Yeh, C.-C. Cheng, Y.-H. Kuo, T.-H. Chen, C.-J. Yang, C.-L. Lin, and C.-C. Wu, "LTPS AMOLED displays incorporating high-contrast top-emitting OLEDs," in Dig. Tech. Papers of 2005 Int. Symp. Soc. of Inf. Display, 2005, pp. 1348-1351.

[71] M. Born and E. Wolf, Principles of Optics, 7th ed. Cambridge, U.K.: Cambridge Univ. Press, 1999, ch. 7.

[72] O. S. Heavens, Optical Properties of Thin Solid Films. New York: Dover, 1991, ch. 7.

[73] B. Masenelli, A. Gagnaire, L. Berthelot, J. Tardy, and J. Joseph, "Controlled spontaneous emission of a tri(8-hydroxyquinoline) aluminum layer in a microcavity," J. Appl. Phys., vol. 85, no. 6, pp. 3032-3037, Mar. 1999.

[74] T. Nakayama, Y. Itoh, and A. Kakuta, "Organic photo- and electroluminescent devices with double mirrors," Appl. Phys. Lett., vol. 63, no. 5, pp. 594-595, Aug. 1993.

[75] A. Dodabalapur, L. J. Rothberg, and T. M. Miller, "Color variation with electroluminescent organic semiconductors in multimode resonant cavities," Appl. Phys. Lett., vol. 65, no. 18, pp. 2308-2310, Oct. 1994.

[76] T. Tsutsui, N. Takada, S. Saito, and E. Ogino, "Sharply directed emission in organic electroluminescent diodes with an optical-microcavity structure," Appl. Phys. Lett., vol. 65, no. 15, pp. 1868-1870, Oct. 1994.

[77] N. Takada, T. Tsutsui, and S. Saito, "Control of emission characteristics in organic thin-film electroluminescent diodes using an optical-microcavity structure," Appl. Phys. Lett., vol. 63, no. 15, pp. 2032-2034, Oct. 1993.

[78] E. Hecht, Optics, 3rd ed. New York: Addison-Wesley Longman Inc., 1998, ch. 9.

[79] Y.-S. Tyan, J. D. Shore, G. Farruggia, and T. R. Cushman, "Broadbandemitting microcavity OLED device," in Dig. Tech. Papers of 2005 Int. Symp. Soc. of Inf. Display, 2005, pp. 142-145.

[80] M. Kashiwabara, K. Hanawa, R. Asaki, I. Kobori, R. Matsuura, H. Yamada, T. Yamamoto, A. Ozawa, Y. Sato, S. Terada, J. Yamada, T. Sasaoka, S. Tamura, and T. Urabe, "Advanced AMOLED display based on white emitter with microcavity structure," in Dig. Tech. Papers of 2004 Int. Symp. Soc. of Inf. Display, 2004, pp. 1017-1019.

[81] R. M. A. Dawson and M. G. Kane, "Pursuit of active matrix organic light-emitting diode displays," in Dig. Tech. Papers of 2003 Int. Symp. Soc. of Inf. Display, 2003, pp. 14-17.
Chung-Chih Wu was born in Tao-Yuan, Taiwan, R.O.C., in 1968. He received the B.S. degree in electrical engineering from National Taiwan University, Taipei, Taiwan, R.O.C., in 1990, and the M.A. and Ph.D. degrees in electrical engineering from Princeton University, Princeton, NJ, in 1994 and 1997, respectively.

From 1990 to 1992, he was an ensign instructor at R.O.C. Naval Communication and Electronics School, Kaohsiung, Taiwan, R.O.C. From 1997 to 1998, he was with the Electronic Research and Service Organization in the Industry Technology Research Institute (ERSO/ITRI), Hsinchu, Taiwan, R.O.C., as a researcher in the division of flat panel display. In 1998, he joined the faculty of National Taiwan University in the Graduate Institute of Electro-optical Engineering and the Department of Electrical Engineering, where he is currently an associate professor. His current research interests include organic semiconductors for optoelectronic and electronic devices, and flat panel displays.

Dr. Wu is the recipient of 2004 Research Award of Academica Sinica, 2003 Ta-You Memorial Research Award of National Science Council, Outstanding Young Electrical Engineer of Chinese Institute of Electrical Engineers, and 2004 Outstanding Innovation Award of ERSO/ITRI, of Republic of China.

Chieh-Wei Chen received the B.S. degree in physics from National Taiwan University, Taipei, Taiwan, R.O.C., in 1999, and the M.A. and Ph.D. degrees in electro-optical engineering from National Taiwan University in 2001 and 2005, respectively.

Since 2005, he has been with AU Optronics Corporation in Hsinchu, Taiwan. His current research interests include organic light-emitting devices (OLEDs) and flat panel displays.

Chun-Liang Lin received the B.S. degree in electrical engineering from National Taiwan University, Taipei, Taiwan, R.O.C., in 2001. He is currently working toward the Ph.D. degree at the Graduate institute of Electro-Optical Engineering, National Taiwan University. His current research interests include organic optoelectronic and electronic devices, optical modeling of optoelectronics, and flat panel displays.

Chih-Jen Yang received the B.S. degree in mechanical engineering from National Taipei institute of Technology in 1993, and the M.A. degree in mechanical engineering from National Taiwan University of Science and Technology in 1998.

He was with RiTdisplay Corporation in Hsinchu, Taiwan, R.O.C., from 1998 to 2003. He is currently working toward the Ph.D. degree at the Graduate Institute of Electro-Optical Engineering, National Taiwan University.

His research interests include semiconductor processing technologies, organic optoelectronic and electronic devices, and flat panel displays. 